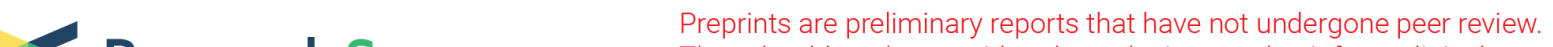 $\begin{array}{ll}\text { Research Square } & \text { They should not be considered conclusive, used to inform clinical practice, } \\ \text { or referenced by the media as validated information. }\end{array}$
}

\section{The Plasmodium falciparum protein PfMRP1 functions as an influx $A B C$ transporter}

\section{Miguel Silva}

Life and Health Sciences Research Institute (ICVS), School of Medicine, University of Minho

\section{Carla Calçada}

Life and Health Sciences Research Institute (ICVS), School of Medicine, University of Minho

\section{Nuno Osório}

Life and Health Sciences Research Institute (ICVS), School of Medicine, University of Minho https://orcid.org/0000-0003-0949-5399

\section{Vitória Baptista}

Life and Health Sciences Research Institute (ICVS), School of Medicine, University of Minho

\section{Vandana Thathy}

Columbia University Medical Center https://orcid.org/0000-0001-7797-8638

\section{Miguel Teixeira}

InnovPlantProtect CoLab (INPP)

\section{Adam Reid}

https://orcid.org/0000-0002-5926-7768

\section{Pedro Ferreira}

Life and Health Sciences Research Institute (ICVS), School of Medicine, University of Minho

\section{David Fidock}

Columbia University Medical Center https://orcid.org/0000-0001-6753-8938

\section{Maria Veiga ( $\nabla$ mariaveiga@med.uminho.pt)}

Life and Health Sciences Research Institute (ICVS), School of Medicine, University of Minho

\section{Article}

Keywords: ABC importer, Plasmodium falciparum, PfMRP1, gene editing, antifolate

Posted Date: January 13th, 2022

DOI: https://doi.org/10.21203/rs.3.rs-1210682/v1

License: (c) (i) This work is licensed under a Creative Commons Attribution 4.0 International License. Read Full License 


\section{Abstract}

Adenosine triphosphate (ATP)-binding cassette (ABC) transporters play an important role in mediating solute or drug transport across cellular membranes. Although this class of transporters has been well characterized in diverse organisms little is known about the physiological roles in Plasmodium falciparum, the deadliest malaria parasite species. We studied the Plasmodium falciparum Multidrug Resistance-associated Protein 1 (PfMRP1; PF3D7_0112200), an ABC transporter localized to the parasite plasma membrane, generating genetic disrupted parasites. We demonstrate that parasites with disrupted pfmrp 1 are resistant to folate analogs, methotrexate and aminopterin, with antimalarial activity. This phenotype occurs due to reduction in compound accumulation in the parasite cytoplasm. Phylogenetic analysis supports pfmrp 1 being distantly related to $A B C$ transporters in other eukaryotes, suggesting an unusual function. We propose that PfMRP1 can act as a solute importer, a function not previously observed in this organism.

\section{Introduction}

Malaria is a major cause of illness throughout the tropical world and is responsible for an estimated 400,000 deaths per year ${ }^{1}$. Treatment and control efforts have been repeatedly hampered by the capacity of Plasmodium falciparum parasites to develop antimalarial drug resistance, making it imperative to understand the molecular basis of resistance and identify new therapeutic targets.

The $A B C$ transporter superfamily is known to transport a wide variety of substrates across cellular membranes against a concentration gradient, an action driven by ATP hydrolysis. These transporters are present in multiple life forms, from prokaryotes to the most complex eukaryotes ${ }^{2}$. ABC transporters also serve as drug targets and are important mediators of drug resistance. The overall architecture of $A B C$ transporters is conserved, with transmembrane domains (TMDs) and nucleotide binding domains (NBDs) that provide energy through ATP hydrolysis. The NBDs are highly conserved and considered the hallmark of the $A B C$ transporter family, while most variation occurs in $T M D s^{3}$. ABC transporters can mediate active influx or efflux of structurally and functionally diverse compounds across membranes, and are classified as importers or exporters, respectively ${ }^{3}$. Importers are present mainly in prokaryotes and plants, and frequently rely on a substrate binding protein (SBP) for recognition and transport of the substrate ${ }^{4-11}$. SBPs can be present in the periplasm or be membrane-bound and interact with TMDs ${ }^{6}$. Exporters are widespread across kingdoms, in prokaryotes and eukaryotes, and do not require SBPs for transport. Various classifications have been employed, such as importers divided into three classes and exporters into two based on their 3D conformation and mechanism of transport ${ }^{2,6}$. Recently $A B C$ transporters have been reclassified based on TMD folds obtained from high-resolution X-ray crystallography and singleparticle cryo-electron microscopy ${ }^{12}$. This new grouping has revealed that transporters of the same type with a similar scaffold can be functionally diverse, acting as importers, exporters, ion channels, or lipid floppases $^{12}$. 
Transporter proteins are key mediators of nutrient uptake or extrusion of waste products and toxic compounds. Due to their capacity to direct substrates between cellular compartments, transporters are frequently associated with drug resistance. The $P$. falciparum genome encodes at least $16 \mathrm{ABC}$ proteins, 11 of which are putative transporters ${ }^{13}$. One of these, P. falciparum Multidrug Resistance Transporter protein 1 (PfMDR1) is a well-known modulator of antimalarial resistance ${ }^{14}$. This transporter is present on the parasite digestive vacuole membrane with ATP-binding loops located on the cytoplasmic side, and transport substrates from the cytoplasm to the vacuole ${ }^{15}$.

Two other ABC transporters, P. falciparum Multidrug Resistance-associated Protein 1 and 2 (PfMRP1 and PfMRP2 ) have been tentatively linked to drug resistance ${ }^{16-22}$. The substrates of these transporters remain largely unexplored, and identification of these could pave the way to developing new antimalarial therapies.

PfMRP1 has been localized at the parasite plasma membrane and intracellular membrane networks ${ }^{13,20}$. Physiological substrates of MRPs in other organisms are amphipathic organic anions like glutathione, glucuronide, or sulfate-conjugated compounds. In $P$. falciparum, PfMRP1 was suggested to function as a potential glutathione or folate transporter ${ }^{20,23-25}$.

Early reports have associated PfMRP1 single nucleotide polymorphisms with different susceptibilities to the antimalarials chloroquine (CQ) and quinine $(\mathrm{QN})^{26}$. Further studies on in vitro drug responses of clinical isolates and in vivo malaria chemotherapy data appeared to support these associations ${ }^{16,17,20,22,27,28}$. However, conflicting reports have emerged while trying to validate the role of PfMRP1 in drug resistance in vitro. One study provided evidence that asexual blood stages parasites that lacked PfMRP1 accumulated more intracellular glutathione, CQ and QN than wild-type (WT) parasites ${ }^{20}$. Knockout parasites also displayed a slightly increased susceptibility to several other antimalarials including piperaquine (PPQ), artemisinin (ART), and primaquine (PMQ) compared with WT parasites ${ }^{20}$. These data suggested that PfMRP1 is involved in the efflux of these drugs and that its absence led to increased intracellular accumulation and therefore more drug reaches the site of action. However, the pfmrp 1 knockout lines showed impaired growth rates, particularly at higher parasitemia, thus confounding any evidence of a direct role of the protein in the drug responses. Other reports demonstrated no impact of a pfmrp 1 knockout on parasite susceptibility to a majority of the antimalarials tested with no apparent fitness $\operatorname{cost}^{25,29}$. These studies differed in their determination of parasite physiological responses, choice of parasite strains, genetic manipulation techniques, and the culture conditions. Nevertheless, this transporter seems dispensable for asexual blood stage development, at least in certain in vitro conditions. Additionally, Rijpma and colleagues showed that parasites with disrupted pfmrp 1 presented a decrease in folate concentration in the parasitized erythrocytes, suggesting a folate exporter function ${ }^{25}$. However, there was no distinction of folate from the parasite versus the erythrocyte in this study. These parasites also displayed decreased sensitivity towards methotrexate (MTX), an antifolate compound and folate analog, but not towards other structurally distinct antifolate compounds such as pyrimethamine (PYR), WR99210 (WR) and trimethoprim (TMP) 25 . 
Applying zinc-finger nuclease (ZNF) gene editing, we herein describe two pfmrp1-disrupted lines in the geographically distinct $P$. falciparum 3D7 and Dd2 parasite strains. Our data disclose PfMRP1 as a potential importer that is likely able to transport folate and related compounds, including drugs related to the folate pathway. To the best of our knowledge, this is the first report of a plasma membrane importer from the $A B C$ transporter family in a non-mammalian eukaryotic organism, aside from plants.

\section{Results}

\section{pfmrp1 disruption}

Using customized ZFNs we disrupted the pfmrp 1 gene in $P$. falciparum Dd2 and 3D7 parasites, by single crossover integration of plasmid into the gene locus (Supplementary figure 1). These two strains originate from Southeast Asia and Africa respectively, are part of genetically distinct sub-populations of $P$. falciparum, and harbor very distinct antimalarial drug responses (Figure 1) ${ }^{30-33}$. ZFNs were engineered to bind neighboring sites on opposite strands of pfmrp1, producing a double-stranded break $1208 \mathrm{bp}$ upstream of the stop codon. Our homology-driven template consisted of a $2.5 \mathrm{~kb}$ pfmrp 1 fragment engineered with three non-synonymous mutations at the ZFN binding site to prevent ZFNs from cleaving the plasmid, and promoted site-specific plasmid integration, thereby disrupting pfmrp1.

Red blood cells (RBCs) infected with ring-stage $P$. falciparum 3D7 or Dd2 parasites were electroporated with the pfmrp1 ZFN disruption plasmid (Supplementary figure 1). This plasmid expresses the blasticidin $S$ deaminase selection marker conferring resistance to blasticidin. Parasite growth was observable by microscopy 18-22 days post drug selection. pfmrp 1 disruption was confirmed by PCR, which identified the full-length gene only in non-mutated WT strains (Supplementary figure 1). PCRs spanning both ends of integration were positive for knockout $(\Delta p f m r p 1)$ strains, denoted $3 \mathrm{D} 7^{\Delta p f m r p 1}$ and Dd2 ${ }^{\Delta p f m r p 1}{ }^{1}$, which were then cloned by limiting dilution (Supplementary figure 1). RNA sequencing analysis (Supplementary figure 2 and data set 1 ) showed that pfmrp 1 was expressed at a lower level in the $\operatorname{Dd} 2^{\triangle p f m r p 1}$ line, confirming that the disruption influenced the expression of the full transcript.

\section{pfmrp1 disruption has no impact on quinoline and dihydroartemisinin susceptibility}

In vitro drug susceptibility assays were performed on WT and $\Delta p f m r p 1$ lines. Tightly synchronized ringstage parasites were incubated for 72 hours with antimalarials, using a range of 10 compound concentrations serially diluted by 2 -fold. Parasitemias were quantiled using Dow cytometry. $\mathrm{IC}_{50}$ values were determined for $C Q$, mefloquine (MQ), lumefantrine (LMF), dihydroartemisinin (DHA), PYR, TMP, WR, MTX and aminopterin (AMT). Of note, there were no alterations in $\mathrm{IC}_{50}$ values for the commonly used quinoline antimalarials $C Q, M Q$ and LMF, nor to $D H A$ for either $3{ }^{2} 7^{\Delta p f m r p} 1$ or $D d 2^{\Delta p f m r p}{ }^{1}$ strains when compared to their parental strains (Figure $1 \mathrm{~A}$ ). $I \mathrm{IC}_{50}$ values were in the range of previous reports. The main differences observed in CQ susceptibility between the parental Dd2 and 3D7 strains are explained by known polymorphisms in the $P$. falciparum chloroquine resistance transporter (PfCRT) and PfMDR1 14,34-36. 
pfmrp1 disruption leads to resistance to folate analogs

${ }_{3 D} 7^{\Delta p f m r p 1}$ and $D d 2^{\Delta p f m r p 1}$ were strikingly less sensitive to the antifolates MTX and AMT, compared to their respective parental strains. The $\mathrm{IC}_{50}$ values for the $3 \mathrm{D} 7^{\Delta p f m r p 1}$ and $\mathrm{Dd} 2^{\Delta p f m r p}{ }^{1}$ lines were increased by 15 and 40 -fold when assayed against MTX $(P<0.0001)$ and 6 and 25 -fold against AMT $(P<0.0001)$, respectively, compared to their WT parental controls (Figures 1A and 1D). However, WT and $\Delta p f m r p 1$ lines displayed comparable responses to the antifolates PYR, TMP and WR. Strain-specific differences in susceptibility to PYR, TMP and WR were observed as expected due to genotypic differences in the $P$. falciparum dihydrofolate reductase (pfdhfr) gene ${ }^{37,38}$. MTX and AMT are folate analogs (Figure 1B), while PYR, TMP and WR are structurally distinct to folate, which might account for the differential pattern of susceptibility changes to these antifolates. These results suggest that loss of pfmrp 1 leads to parasite resistance to folate analogs. This would be incompatible with an exporter function, as the disruption should lead to increased drug concentration in the parasite cytoplasm leading to heightened drug sensitivity in the $\triangle p f m r p 1$ lines. These findings suggest PfMRP1 as an importer present in the $P$. falciparum plasma membrane.

To confirm that the observed resistance phenotype was mediated by PfMRP1, the MRP chemical inhibitor MK571 was used to block PfMRP1 transport in the Dd2 strain ${ }^{39-41}$. A small but significant increase (1.3fold, $\mathrm{P}<0.001)$ in $\mathrm{IC}_{50}$ was observed for MTX only in MK571-treated Dd2 WT parasites $(24.7 \pm 1.4 \mathrm{nM})$ compared to untreated controls $(18.8 \pm 0.8 \mathrm{nM})$, whereas no difference was observed for the MK571treated Dd2 ${ }^{\triangle p f m r p} 1$ line, supporting a PfMRP1-mediated effect (Figure $1 \mathrm{C}$ ). The fold-increase in MTX IC 50 values by chemical inhibition was considerably lower compared to the increase observed with the $\mathrm{Dd}^{\triangle p f m r p} 1$ line (Figures $1 \mathrm{~A}$ and 1D), which might be explained by incomplete inhibition of PfMRP1mediated transport by MK571.

We next examined the effect of inhibiting PfCRT and PfMDR1 in Dd2 ${ }^{\triangle p f m r p} 1$ parasites with verapamil and elacridar, respectively, to understand the possible crosstalk between these antimalarial drug resistance mediators and PFMRP1. Verapamil-mediated inhibition led to a small $(1.2$-fold, $P<0.01)$ but significant decrease in MTX susceptibility in Dd2 ${ }^{\Delta p f m r p 1}$ parasites ( $896 \pm 42.4 \mathrm{nM}$ compared to $767 \pm 42.4 \mathrm{nM}$ ), whereas no effect was observed in Dd2 WT (Supplementary Figure 3). These results suggest that PfCRT might interplay with PfMRP1 to augment MTX resistance (Supplementary Figure 3). This increased resistance could be due to trapping of residual MTX in the digestive vacuole, away from its cytosolic site of action, with MTX being unable to be effluxed by PfCRT in the presence of verapamil that is a known blocker of PfCRT-mediated drug transport ${ }^{42}$. No effect was observed with elacridar-mediated inhibition (Supplementary Figure 3).

\section{pfmrp1 -disrupted parasites accumulate less fluorescein methotrexate}

To understand how resistance to folate analogs occurs in the $\Delta p f m r p 1$ parasites, we measured the timedependent accumulation of fluorescein MTX (F-MTX), a fluorescent derivative of MTX, using flow 
cytometry. Remarkably, the Dd2 $2^{\Delta p f m r p} 1$ line showed a significant lack of F-MTX accumulation over time, with an average of 3.3 times less F-MTX fluorescence intensity after 3 hours of incubation compared to Dd2 WT parasites $(P<0.01)$ (Figure $2 A)$. The observed F-MTX phenotype seems specific to PfMRP1mediated transport (Figure 2B). Fluo-4 is a calcium probe that enters the parasite through passive diffusion, accumulates in the parasite digestive vacuole, and can be modulated by PfMDR1-mediated transport ${ }^{43,44}$. Repeating the assay with Fluo-4 showed no differences in accumulation between Dd2 WT and $\Delta p f m r p 1$ parasites (Figure 2B). These results provide evidence that PfMRP1 can import F-MTX, an effect that is much reduced in $\mathrm{Dd} 2^{\triangle p f m r p 1}$, thus explaining the resistance phenotype. The residual accumulation observed in $\mathrm{Dd} 2^{\triangle p f m r p 1}$ might occur through a distinct transport pathway, passive diffusion, or both.

To confirm the location of F-MTX inside the infected RBC, parasites were incubated with F-MTX and visualized using confocal microscopy. After a 3-hour incubation period, F-MTX accumulated in the parasite cytosol, co-localizing with MitoTracker fluorescence that labels mitochondria, but not with the DAPI nuclear stain (Figure 2C). Confocal microscopy confirmed the same accumulation pattern observed in the flow cytometry assay, with Dd2 WT exhibiting significantly more F-MTX accumulation than $\mathrm{Dd}_{2}{ }^{\Delta p f m r p 1}$ (Figure $2 \mathrm{C}$ ). The fluorescence ratio of F-MTX/MitoTracker demonstrated significantly higher $(P<0.0001)$ F-MTX accumulation in Dd2 WT parasites (Figure 2D).

However, a drug susceptibility assay showed that F-MTX does not retain the antimalarial activity of MTX, most likely due its bulkier structure preventing binding to PfDHFR, the target of MTX (Supplementary Figure 4). Since transport proteins are less specific in their substrate binding properties compared to the interaction of a substrate with the less accessible active site of an enzyme, F-MTX might be transported into the parasite cytoplasm via PfMRP1 but unable to bind PfDHFR and impair parasite growth.

Overall, both the flow cytometry and confocal microscopy data on the transport of F-MTX into infected RBCs point to a putative import function of PfMRP1.

\section{Folate analogs compete for PfMRP1 transport}

To further explore the propensity of PfMRP1 to transport antimalarial drugs, the F-MTX probe was used as a proxy of transport capacity. We performed flow cytometry competition assays and measured the fluorescence of F-MTX in infected RBCs, after 3 hours incubation periods with varying concentrations of different compounds and a fixed concentration of F-MTX. These assays tested CQ, MQ, DHA, and amodiaquine (AQ), as well as the antifolates MTX, AMT, PYR, TMP and MK571. Competition assays showed that increases in the MTX, AMT or MK571 concentration caused concentration-dependent decreases in F-MTX accumulation in Dd2 WT parasites but not in $\Delta p f m r p 1$ parasites (Figure 3 ). These results demonstrate that F-MTX competes for the same transport pathway as MTX, AMT and MK571. This pathway is mediated by PfMRP1, as evidenced by the lack of competition in the Dd2 ${ }^{\triangle p f m r p} 1$ line (Figure 3). These assays further suggest that residual F-MTX in the Dd2 ${ }^{\Delta p f m r p 1}$ strain is probably the result of another transport mechanism or passive transport. The reduction in accumulation was more 
pronounced for MTX and AMT compared to MK571. This finding is likely related to MK571 acting as a weak chemical inhibitor of PfMRP1, as supported by the $\mathrm{IC}_{50}$ results in the presence of MK571 (Figure 1C), compared to PfMRP1 role in direct transport of MTX and AMT.

\section{Folate impacts parasite growth}

Parasite growth was monitored to understand the overall impact of $\Delta p f m r p 1$. Regular levels of folate in human serum are around 6-20 $\mu \mathrm{g} / \mathrm{L}$ and folate deficiency in RBCs is considered below $151 \mu \mathrm{g} / \mathrm{L}^{45}$. Standard RPMI medium used for in vitro culture contains $1 \mathrm{mg} / \mathrm{L}$ folate, which is 10 times higher than the limit of folate deficiency in RBCs and around 50 times higher than in human serum. Therefore, we modified an assay to limit exogenous folate in order to reduce parasite folate pools and evaluate parasite reliance on external folate sources, and hence PfMRP1 dependency ${ }^{38}$. Additionally, since $\Delta p f m r p 1$ impacts folate-related transport, growth was evaluated in culture medium lacking an exogenous source of folate. Parasites were cultured for 14 days in medium depleted of folate, after which parasites were tightly synchronized at $0.05 \%$ parasitemia and growth was measured in regular medium, medium depleted of folate or medium containing $100 \mu \mathrm{g} / \mathrm{L}$ folate. Both $3 \mathrm{D}^{\Delta p f m r p 1}$ and Dd2 ${ }^{\Delta p f m r p 1}$ parasites were able to achieve high parasitemias like WT control parasites under regular culture conditions, although $D d 2^{\Delta p f m r p 1}$ displayed slightly less growth at high parasitemias ( $>5 \%$ ) (Figure $4 \mathrm{~A}$ ). Without exogenous folate supplementation the $\mathrm{Dd} 2$ strain displayed similar growth in the first cycle but slower growth rate in the second cycle, with around $2 \%$ parasitemia compared to $6-8 \%$ of regular culture medium. Dd2 parasites were nonetheless able to reach parasitemias of around $8 \%$ one cycle later (Figure 4A). In contrast, 3D7 parasites displayed similar growth rates in medium with or without exogenous folate (Figure 4B). There was no difference in growth between WT and $\Delta p f m r p 1$ strains in medium without exogenous folate (Figure 4A and $4 B$ ). To confirm that this was not an artifact due to parasites still having access to folate pools, cultures were diluted multiple times using RBCs from the same blood donor bag and growth was monitored in medium lacking folate ${ }^{46}$. Results showed no difference between WT and $\Delta p f m r p 1$ growth for both strains in continuous culture in medium depleted of folate (Figure 4C and 4D). Overall, no differences in growth between WT and $\Delta p f m r p 1$ were observable for both 3D7 and Dd2 strains.

The assay with medium containing $100 \mu \mathrm{g} / \mathrm{L}$ folate had results comparable to medium without folate. Addition of a source of exogenous folate partly recovered Dd2 growth compared to medium without folate, with parasites able to grow to $4 \%$ on the second cycle compared to $2 \%$ in medium without folate (Figure 4A). As with medium lacking folate, there was no impact on the 3D7 growth rate (Figure 4B). These results support the impact of exogenous folate on the growth rate of $D d 2$, which we observed to be considerably faster than 3D7 under our in vitro conditions. Accordingly, the Dd2 growth rate without folate supplementation resembled the 3D7 growth rate (Figure 4A and 4B). Although, PfMRP1 might impact parasite growth as observed for $\mathrm{Dd} 2^{\Delta p f m r p 1}$ cultured in standard medium, likely this impact is only observable under very rich in vitro growth conditions or as a result of fitness cost at high parasitemia as 
previously reported ${ }^{20}$. Furthermore, the effect of folate on the overall Dd2 growth is likely independent of PfMRP1 and mediated by alternative pathways.

\section{Folic acid impacts the antifolate response}

To understand the impact of folate on the antifolate resistance phenotype observed in $\Delta p f m r p 1$ parasites, we performed 72-hour drug susceptibility assays under various folate conditions. $I_{50}$ values were measured for PYR, TMP, WR, AMT, MTX and the two non-antifolate based compounds ferroquine and DMS265, as controls. Assays were performed in the presence of diminished folate conditions, namely no exogenous folate, and a folate supplementation of $3 \mu \mathrm{g} / \mathrm{L}$ or $100 \mu \mathrm{g} / \mathrm{L}$ folic acid, the last being the folic acid concentration previously tested and that partly recovers $\mathrm{Dd} 2$ growth rate. Similar to growth assays (Figure 4) there was no impact on the overall growth of these strains in the 72-hour period of the assay. Results showed a slight but significant increase in susceptibility of Dd2 ${ }^{\Delta p f m r p}{ }^{1}$ relative to WT parasites when assayed against PYR or TMP, relative to WT parasites, under conditions of no folate or $3 \mu \mathrm{g} / \mathrm{L}$ of folate, respectively, and $100 \mu \mathrm{g} / \mathrm{L}$ of folate (Figure 5 ). These results suggest that an inefficiency of $\mathrm{Dd} 2^{\Delta p f m r p 1}$ to import folate could render the drugs slightly more effective under limiting exogenous folate conditions, possibly due to lower intracellular dihydrofolate levels, the natural substrate of PfDHFR. Other folate conditions for PYR, TMP, and WR did not show significant differences but presented the same trend. An inefficiency to import folate could further explain the phenotype of the decreased growth of $\mathrm{Dd} 2^{\Delta p f m r p 1}$ under regular conditions (Figure 4)

The resistance phenotype of $3 \mathrm{D}^{\Delta p f m r p 1}$ and $\mathrm{Dd} 2^{\Delta p f m r p 1}$ parasites for MTX and AMT was maintained under all folate conditions (Figure 5). Surprisingly, the susceptibility to AMT was drastically reduced under lower folate concentrations, an effect that was even more pronounced for the $3 \mu \mathrm{g} / \mathrm{L}$ folic acid supplementation compared with no exogenous folate (Figure 5). A similar effect was observed for MTX, although with a less pronounced difference and evident only in the Dd2 ${ }^{\Delta p f m r p} 1$ and parental Dd2 lines (Figure 5). This phenotype might be multifactorial, as it seems to be independent of PfMRP1 for AMT, as the effect is observable also in WT parasites. A slight increase in $\mathrm{IC}_{50}$ was also observed for Dd2 WT at 3 $\mu \mathrm{g} / \mathrm{L}$ folic acid for MTX (2-fold, $\mathrm{P}<0.01$ ), additionally pointing to a PfMRP1-independent effect. These data, with $\mathrm{Dd} 2$ drug response being more affected by folate concentration agree with parasite growth assays, in which Dd2 was generally more impacted by folate concentrations.

Both MTX and AMT are expected to be competitors of folate for the PfDHFR binding site, and in this case a lower folate concentration was expected to render parasites more susceptible to drug action. However, the opposite was observed where less exogenous folate led to lowered susceptibility to the antifolate analogs MTX and AMT. This result suggests that this phenotype was likely independent of PfMRP1 and was instead due to another mechanism. Likewise, a similar phenotype was observed for antifolates that are structurally different from folate (PYR, TMP, WR) and even unrelated compounds that are not expected to act on the folate pathway (DSM265 and ferroquine) (Figure 5). Overall, this phenotype was only observed in the Dd2 strain, except for AMT where this was also observed in the 3D7 strain. 
pfmrp1 is phylogenetically unrelated to other eukaryotic ABC transporters

$A B C$ transporters are an ancient family present in a wide variety of species ranging from prokaryotes to eukaryotes. The results suggesting that PfMRP1 can function as an influx ABC transporter prompted us to investigate evolutionary relationships among PfMRP1 and other proteins from the same family and explore potential links between sequence similarity and function. We started by using the PfMRP1 amino acid sequence to BLASTp query the landmark database that includes a taxonomically diverse and nonredundant set of protein sequences. This resulted in 116 protein hits from 11 different species (Caenorhabditis elegans, Arabidopsis thaliana, Dictyostelium discoideum, Glycine max, Saccharomyces cerevisiae, Drosophila melanogaster, Danio rerio, Homo sapiens, Schizosaccharomyces pombe, Mus musculus and Leishmania donovani) (Supplementary dataset 2). Except for PfMRP2 that shared moderate sequence identity with PfMRP1 (49\% identity and 64\% query coverage), all hits from other organisms showed low identity $(<25 \%)$. Phylogenetic analysis using these sequences placed PfMRP1 and PfMRP2 in an isolated branch in the phylogeny (Figure 6). These sequences were inferred to share a node, deep in the phylogeny, only with an ABC transporter from Leishmania donovani (XP_003863220.1) but with a low bootstrap support value. In contrast, several other $A B C$ transporters from different species were found to be highly related to each other branching within well supported monophyletic clades. This finding suggests that although PfMRP1 harbors transmembrane helices typically found in the ABC transporters that function as exporters, it has a distinct protein sequence and origin. This finding is compatible with the hypothesis that PfMRP1 might have an atypical transport function.

\section{Discussion}

The ABC superfamily is the largest transporter protein family present in virtually all organisms. In nonmammalian eukaryotes, apart from plants, the $A B C$ superfamily has only been reported to comprise of exporter proteins. This family has been linked to protecting cells against toxic agents, including playing a role in drug resistance phenotypes. PfMRP1 is an ABC transporter whose biological role remains elusive in $P$. falciparum. pfmrp 1 has been suggested to be under natural selective drug pressure but no compelling evidence has shown this transporter to mediate a resistance phenotype ${ }^{16,17,26,47}$. Our study of genetically engineered $P$. falciparum lines with disrupted pfmrp 1 in two different genetic backgrounds, derived from Africa and Asia phylogeographic sub-populations, supports that PfMRP1 does not substantially impact the in vitro susceptibility to commonly used antimalarials such as CQ, DHA, LUM, and MQ (Figure 1). Moreover, this transporter is dispensable for asexual blood stage parasite growth, provided the right in vitro conditions.

Interestingly, in agreement with an earlier report ${ }^{25}$, we found that $\Delta p f m r p 1$ provided markedly increased resistance to the folate analog MTX, but not to other structurally unrelated antifolates such as PYR, WR or TMP. Additionally, we observed that $\triangle p f m r p 1$ also provides resistance to AMT, another folate analog, pinpointing this phenotype to be specific for folate-related compounds. Using MK571, a MRP inhibitor ${ }^{40}$, we observed a decrease in MTX susceptibility only in the WT strain and not in $\Delta p f m r p 1$ parasites, demonstrating this phenotype to be specific for PfMRP1 (Figure 1). The lowered sensitivity to folate 
analogs observed in the $\Delta p f m r p 1$ lines suggests reduced drug accumulation in the parasite cytoplasm where the target enzyme PfDHFR is located. Once in the cytoplasm, the antifolates compete with folate for binding to the PfDHFR active site, thereby inhibiting the folate pathway by blocking reduction of dihydrofolic acid to tetrahydrofolic acid, a key cofactor used in carbon transfer reactions. Using labelled fluorescein F-MTX we showed through microscopy and flow cytometry, that F-MTX accumulates in the cytoplasm of WT parasites in contrast to $\triangle p f m r p 1$ parasites, which only showed residual F-MTX accumulation (Figure 2). This suggests that in WT parasites PfMRP1 is responsible for transporting FMTX and likely other folate analogs into the parasite cytosol.

MRP proteins are folate transporters in diverse organisms, and in humans they are associated with transport of the anticancer agent MTX ${ }^{48-50}$. In P. falciparum, PfMRP1 has been associated with folate transport in vitro and in vivo ${ }^{17,25}$. Previous pfmrp 1 knockout experiments suggested apparently contradictory results of PfMRP1-mediated export of folate from the parasite cytoplasm, while $\Delta p f m r p 1$ parasites displayed reduced susceptibility to the antifolate $M T X^{25}$. Due to the proposed exporter function of PfMRP1, this resistance phenotype was hypothesized to result from increased folate accumulation within the $\Delta$ pfmrp 1 parasites that could compete with MTX due to lack of export. However, our data, including drug assays with varying concentrations of folate, demonstrated that folate supplementation within the culture medium increases susceptibility to a panel of antimalarials. This included the folate analogs MTX and AMT, making it unlikely that folate competition is the main driver of the observed resistance phenotype (Figure 5). Another proposed hypothesis based on structural similarity is that PfMRP1 directly transports MTX ${ }^{25}$. Under the new proposed importer function, a decrease in cytoplasmic MTX concentration in $\triangle p f m r p 1$ parasites due to the absence of PfMRP1-mediated transport would explain reduced susceptibility. Our data using labelled MTX supports the direct MTX transport hypothesis, implicating PfMRP1 as an importer protein.

Earlier studies suggested that the main folate uptake pathway in $P$. falciparum is dependent on the proton gradient maintained by proton symporters at the parasite plasma membrane. The ATPase inhibitor concanamycin A greatly reduced folate uptake, linking folate transport with ATP-powered proton transport ${ }^{51,52}$. Considering our results, the mechanism of concanamycin A-mediated inhibition of folate uptake would also be compatible with blockage of the ATP-dependent PfMRP1 transporter potentially involved in parasite folate import. Moreover, MTX has been shown to reduce folate import suggesting a similar pathway of uptake ${ }^{52}$. Accordingly, our competition assays indicate that MTX and AMT are transported by PfMRP1. Nevertheless, although folate, MTX and AMT share very similar structures and are likely to share the same transport pathway, we could not directly demonstrate that folate competes with F-MTX for PfMRP1-mediated transport (Figure 3). P. falciparum is cultured in RBCs that contain folate from the host, thereby making the folate competition assay challenging to interpret since the folate import system could be saturated even without exogenous supplementation of folate. Furthermore, there was no major impact on parasite growth in folate-depleted medium. 
Our growth assays suggest that 3D7 is less dependent on an external folate source compared to Dd2. Since, the lack of folate was shown to reduce the Dd2 parasite growth rate, which in regular conditions is comparably higher to 3D7 (Figure 4). This could possibly be explained by the presence of paraaminobenzoic acid in the medium, which can be used for de novo folate synthesis and compensate for the lack of folate import. P. falciparum synthesizes folate de novo, and as such this pathway might be more functional in 3D7. The higher Dd2 growth rates, with folate supplementation, indicates additional mechanisms to import or use exogenous folate independently of PfMRP1, as $\Delta p f m r p 1$ lines were not differentially impacted by the lack of exogenous folate (Figure 4). The $\Delta p f m r p 1$ impact in Dd2 that causes slightly slower growth than WT, on regular culture conditions, might be related to the fact that this strain is more susceptible to the absence of folate. Consequently, less folate import due to $\Delta p f m r p 1$ might render the parasite less fit.

The PfMRP1 import properties herein observed are not commonly observed in many eukaryotic organisms. Recently, a novel structure of an E. coli ABC importer, YbtPQ, was described that has an ABC type IV exporter structure ${ }^{10}$. This transporter shows significant sequence similarity to Sav1866, a homolog of multidrug $A B C$ transporters including $M D R 1^{10}$. Different $A B C$ classifications are currently in use, including newer groupings based on the more translatable TMD folds and topologies resulting from advances in cryo-electron microscopy and X-ray crystallography ${ }^{12}$. However, even structural reclassification does not enable predictions of transporter functionality. For example, type IV ABCs share a single structural scaffold yet have great functional diversity comprising of importers, exporters, lipid floppases, ion channels and regulators. PfMRP1 is predicted to be in the same structural class as the exporters Sav1866 and MDR1, but also importers such as YbtPQ and ABC Subfamily D Member 4 $(A B C D 4)^{16,53}$. Generally, this scaffold, which includes PfMRP1, allows a wide range of functions across multiple organisms. Obtaining the crystal structure of PfMRP1 would help to mechanistically explore its transporter properties.

Horizontal transfer has played a role in the evolution of apicomplexan and evidence of prokaryote horizontal transfer has been found for the peroxiredoxins, a family of thiol-dependent hydroperoxidases that function as detoxifying enzymes, redox sensors and chaperones ${ }^{54,55}$. Conceivably, the PfMRP1 transporter could also have resulted from horizontal transfer ${ }^{56-58}$. Based on our phylogenetic analysis pfmrp 1 and $p f m r p 2$ have identifiable $A B C$ sequence motifs but have low overall sequence identity with the ABCs of other eukaryotic organisms and thus a distinct origin. Moreover, $P$. falciparum, as an intracellular parasite, encodes far fewer enzymes and transporters compared to other free-living eukaryotic microbes, which might lead the available transporters to have less substrate specificity and wider functionality ${ }^{59}$.

Overall, our data provide evidence that PfMRP1 functions as an ABC importer protein that can mediate import of folate analogs into the parasite cytoplasm. As one of the few $A B C$ importers identified to date in the plasma membrane of a eukaryotic organism, this study highlights the importance of functional studies to elucidate the molecular basis of importer function in $P$. falciparum. 


\section{Materials And Methods \\ Plasmid construction}

Plasmid for pfmrp1 disruption was constructed using a starting pDC2-based vector. CompoZr ${ }^{\circledR}$ Custom ZFNs were purchased from Sigma-Aldrich (St. Louis, Missouri, USA) to induce a double-stranded break in the $3^{\prime}$ end of the pfmrp 1 coding sequence. Two ZFNs (left and right), binding to adjacent sequences on opposite strands of the DNA helix, are required to induce the double-strand break. These were supplied on separate plasmids. The ZFN pair was designed to bind to the sequence

TTATGCATCCTCATT tatatgCAAGTGGAATAA (the DNA cut site is shown in lower case letters and is situated $1208 \mathrm{bp}$ upstream of the pfmrp 1 stop codon). The plasmid encoding the right ZFN also encoded the 2A 'ribosome skip' peptide, which enables polycistronic expression of 2A-linked genes. The two plasmids encoding the ZFNs were digested with Bglll and Xhol and combined to yield an intermediate plasmid with 2A-linked ZFN sequences. The ZFN fusion was then digested with Nhel and Xhol and subcloned downstream of the calmodulin (PF3D7_1434200) promoter and upstream of the $h s p 86$ (PF3D7_0708500) 3' UTR in a pDC2-based vector with the human dihydrofolate reductase (hdhfr)

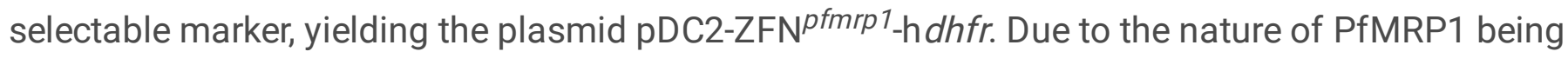
associated with folate transport we decided to change the hdhfr selectable cassette, which codes for the human enzyme that bypasses the action of $P$. falciparum antifolate drugs. The selection cassette was then changed from hdhfr to blasticidin $S$ deaminase gene (bsd). bsd was introduced using Apal and Sacll restriction to yield the plasmid pDC2-ZFN ${ }^{\text {pfmrp } 1}$ _pfmrp 1-bsd. Figure 1A shows the ZFN-mediated pfmrp1 double-stranded break (thunderbolt) that was repaired by recombination with the $p f m r p 1$ homologous region on the plasmid leading to single-crossover plasmid introduction and pfmrp 1 disruption. A $2459 \mathrm{bp}$ donor sequence, encompassing from the pfmrp 1 coding sequence from nucleotides 2999 to 5455 , was amplified from Dd2 genomic DNA with the primers 5'-

GTAGCATATGGTGCTTATTTCGTAAAAGGGAATACGGAGAG and 5'-

GTTGACGTCTAACAAATGTGATAATTCAGATTGCG and inserted into the BstAPI and Aatll sites of pDC2-

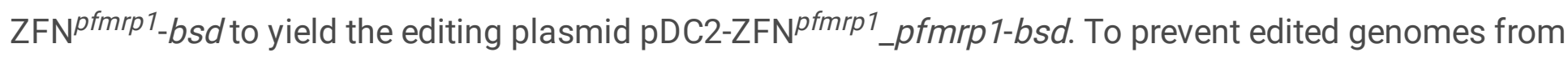
being further cleaved, three silent mutations were engineered into the donor sequence at the ZFN-binding site via site-directed mutagenesis (QuikChange Multi SDM Kit; Agilent Technologies, Santa Clara, California, USA) using the primers $5^{\prime}$ -

ACCCACACTTATATGCTTCTGGTATAATCAAATTATATAAAGAAAAAAATTATG and 5'-

TACCAGAAGCATATAAGTGTGGGTGCATAATAATGTAAGTAGTTAATAAC

\section{Parasite culture and transfection}

P. falciparum 3D7 and Dd2 strains (MRA-120 and MRA-156, MR4-Malaria Resources) were maintained at $4 \%$ hematocrit in RBCs with RPMI-1640 (Gibco) supplemented with $2 \mathrm{~g} / \mathrm{L}$ sodium bicarbonate, $2 \mathrm{mM} \mathrm{L-}$ glutamine, $25 \mathrm{mM}$ HEPES (Gibco), $25 \mu \mathrm{g} / \mathrm{mL}$ gentamycin (Gibco), $100 \mu \mathrm{M}$ hypoxanthine (Sigma-Aldrich) and $0.25 \%(\mathrm{w} / \mathrm{v})$ AlbuMAXII (Gibco) with daily medium changes. Parasites were maintained at $37^{\circ} \mathrm{C}$ in an airtight environment with $3 \% \mathrm{O}_{2}, 5 \% \mathrm{CO}_{2}$ and $92 \%$ of $\mathrm{N}_{2}$. 
Ring-stage cultures at 5\% parasitemia were washed in 1X Cytomix $\left(10 \mathrm{mM} / \mathrm{L} \mathrm{K} \mathrm{HPO}_{4} / \mathrm{KH}_{2} \mathrm{PO}_{4}, 120\right.$ $\mathrm{mM} / \mathrm{L} \mathrm{KCl}, 0.15 \mathrm{mM} / \mathrm{L} \mathrm{CaCl}_{2}, 5 \mathrm{mM} / \mathrm{L} \mathrm{MgCl} 2,25 \mathrm{mM} / \mathrm{L} \mathrm{HEPES}, 2 \mathrm{mM} / \mathrm{L}$ EDTA, adjusted with $10 \mathrm{M} / \mathrm{L} \mathrm{KOH}$ to $\mathrm{pH}$ 7.6) and electroporated with $50 \mu \mathrm{g}$ of purified plasmid DNA eluted in 1X Cytomix, using Gene Pulser®/MicroPulser ${ }^{\mathrm{TM}}$ Electroporation Cuvettes, $0.2 \mathrm{~cm}$ gap (BioRad). The electroporation was performed at $0.31 \mathrm{kV}$ with capacitance of $950 \mu \mathrm{fD}$ on a Gene Pulser Xcell ${ }^{\mathrm{TM}}$ (BioRad) electroporator.

To enable transient expression of ZFN and homology-directed repair and pfmrp 1 disruption, we applied $2.5 \mu \mathrm{g} / \mathrm{mL}$ blasticidin S HCl (Sigma-Aldrich) to the transfected cultures 24 hours post electroporation for 5 days. After selection, parasites were cultured without drug. After microscopic growth was observed, the cultures were genotyped by PCR and tested for gene disruption. Amplification was performed with Phusion Blood Direct PCR Master Mix (ThermoFisher) using $0.5 \mu \mathrm{M}$ of each primer and $1 \mu \mathrm{L}$ of blood from transfected cultures, in a $10 \mu \mathrm{L}$ reaction. P1- 5'- AAGGACATATTTATTAAACCGCAGAG P2- 5'GCATGGGTGTGTGTAAGTTT P3- 5'- CCTTCAAAAATGTTTAATGGATGAT P4- 5'- CTCATGGTTATGGCAGC P5- 5'- TGTGTTAATTTGTGATTCCA (Supplementary figure 1). PCR reactions were performed for 35 cycles with primer annealing at $55^{\circ} \mathrm{C}$ and extension at $62^{\circ} \mathrm{C}$ for 30 seconds per $1 \mathrm{~kb}$ of amplicon. The cultures were subjected to another similar round of selection if the PCR was negative, or the mutant population was present in minimal quantities. Positive bulk cultures were cloned by limiting dilution and positive wells were detected and genotyped by PCR. One clone from each strain was selected to proceed to further experiments.

\section{In vitro antimalarial drug assays}

Drug susceptibility assays were performed using a flow cytometry-based approach described previously, with minor modifications ${ }^{60}$. Assays were performed in flat-bottom 96 well plates in $200 \mu \mathrm{L}$ of culture medium, $1 \%$ hematocrit and $0.3 \%$ starting parasitemia. Assays using reduced folate were performed using RPMI without folate (ThermoFisher, 27016021) and supplemented with $3 \mathrm{ng} / \mathrm{mL}$ or $100 \mathrm{ng} / \mathrm{mL}$ of folic acid (Sigma, F8758). Aaof test compound, serially diluted two-fold. Compounds used were chloroquine (Sigma), mefloquine (Sigma), lumefantrine (Sigma), dihydroartemisinin (Sigma), pyrimethamine (Sigma), trimethoprim (Sigma), WR (Jacobus Pharmaceuticals), methotrexate (Sigma) and aminopterin (Sigma). Two wells were left without drug as growth control. After 72h, parasitemia was assessed in the flow cytometer. To perform drug assays with transporter protein inhibitors these were added at a fixed concentration to all wells: verapamil (Sigma) was used at $100 \mathrm{nM}$, elacridar (Sigma) at $800 \mathrm{nM}$ and MK-571 (Sigma) at $1600 \mathrm{nM}$. For the flow cytometry analysis $60 \mu \mathrm{L}$ of each well from drug assay was incubated with $40 \mu \mathrm{L}$ of staining solution [2X SYBR ${ }^{\text {TM }}$ Green I Nucleic Acid Gel Stain (Invitrogen) and $1.6 \mu \mathrm{M}$ MitoTracker ${ }^{\mathrm{TM}}$ Deep Red FM (Invitrogen)] for 30 minutes at $37^{\circ} \mathrm{C}$. After the incubation, the cells were diluted in $300 \mu \mathrm{L}$ of PBS and assayed on a LSRII flow cytometer (BD Biosciences). SYBR Green strains DNA (parasitized cells) and MitoTracker stains based on mitochondrial membrane potential (live parasites). Data was analysed using FlowJo ${ }^{\mathrm{TM}} \mathrm{V} 10$ software (Becton, Dickinson and Company). Gating was done on the SYBR Green and MitoTracker positive cells giving the parasitemia. Assays were performed with 3 to 10 independent replicates. Statistical evaluations comparing strains were performed using two-tailed unpaired $t$-tests. 


\section{Fluorescein methotrexate accumulation assay and microscopy}

Accumulation of fluorescein methotrexate (F-MTX) (Invitrogen, M1198MP) was measured over time using flow cytometry and microscopy. Parasites were incubated in a hematocrit of $4 \%$ at around $2-4 \%$ parasitemia with $1000 \mathrm{nM}$ F-MTX. Conditions without F-MTX or with uninfected RBC were used as controls for baseline and autofluorescence.

For the flow cytometry analysis 1.6 $\mu \mathrm{M}$ MitoTracker ${ }^{\text {TM }}$ Deep Red FM (Invitrogen)] was incubated with FMTX. After the incubation, at the time points 1-hour, 2-hours and 3-hours, the cells were diluted in $300 \mu \mathrm{L}$ of D-PBS and run on a LSRIl flow cytometer (BD Biosciences). Another condition was performed at 3hours with parasites similarly incubated with Fluo-4 (Invitrogen), instead of F-MTX. Data were analyzed using FlowJo ${ }^{\text {TM }}$ V10 software (Becton, Dickinson and Company). Gatings were performed on the MitoTracker positive cells to determine parasitemias. Mean Alexa Fluor 488 (excitation: 490/emission: 525) fluorescence of live parasites was calculated and plotted. Assays were performed in triplicate and representative plots are presented.

For the microscopy analysis the parasites were collected after a 3-hour incubation time point and further stained for 5 minutes with DAPI, in addition to MitoTracker. Parasites were centrifuged at $1500 \mathrm{rpm}$ for 5 minutes and a microscopic slide of the RBC culture pellet was made for live fluorescent imaging using an Olympus LPS Confocal FV3000 microscope. Fluorescence was quantified using ImageJ software (NIH) by circling the parasite area. 10 parasites were counted per condition, and statistical evaluations were performed using two-tailed unpaired t-tests.

\section{Competition assays}

F-MTX competition assays were performed using a flow cytometry-based approach. The assay was performed in 96-well plates with pre-dosed descending concentrations of test compound, two-fold dilution each consecutive well, with the highest compound concentration of $2000 \mathrm{nM}$. Two wells were left without drug as negative control. All wells were also pre-dosed with a fixed $1000 \mathrm{nM}$ of F-MTX, including controls. In the last 30 minutes of the assay parasites were incubated with 1.6 $\mu \mathrm{M}$ MitoTracker ${ }^{\mathrm{TM}}$ Deep Red FM (Invitrogen)]. After 4 hours, the cells were diluted in $300 \mu \mathrm{L}$ of D-PBS and processed on a LSRII flow cytometer (BD Biosciences). Data were analyzed using FlowJo ${ }^{\mathrm{TM}} \mathrm{V} 10$ software (Becton, Dickinson and Company). Gatings were performed as described above. Assays were performed at least in duplicates.

\section{Growth assays}

Assays were performed in flat-bottom 6 -well plates at $4 \%$ hematocrit and $0.05 \%$ starting parasitemia. Previous to starting the assay, parasites were cultured in RPMI without folic acid (Sigma) for 14 days to reduce possible folate pools derived from culture medium. Parasites were grown under normal culture conditions or in RPMI without folic acid. Moreover, supplementation of medium without folic acid was 
done with addition of $100 \mu \mathrm{g} / \mathrm{L}$ of folic acid (Sigma, F8758). Every 24 hours, parasitemia was assessed in the flow cytometer. Flow cytometry was performed incubating $20 \mu \mathrm{L}$ of each culture with $40 \mu \mathrm{L}$ of staining solution [2X SYBR ${ }^{T M}$ Green I Nucleic Acid Gel Stain (Invitrogen) and 1.6 $\mu$ M MitoTracker ${ }^{T M}$ Deep Red FM (Invitrogen)] for 30 minutes at $37^{\circ} \mathrm{C}$. After the incubation, the cells were diluted in $300 \mu \mathrm{L}$ of PBS and examined on a LSRII flow cytometer (BD Biosciences). SYBR Green stains DNA (parasitized cells) and MitoTracker detects the mitochondrial membrane potential (live parasites). Data were analyzed using Flow Jo ${ }^{\text {TM }}$ V10 software (Becton, Dickinson and Company). Gatings were performed as described above with an additional gate for SYBR Green positive cells.

\section{Transcriptome sequencing and analysis}

Parasites were tightly synchronized with $5 \%$ sorbitol in intervals of 20 hours for 3 consecutive life cycles. RNA was extracted from trophozoite stages, estimated between 28 to 32 hours old, using a RNeasy Mini Kit (Qiagen). Libraries and sequencing were outsourced (Macrogen Inc), using TruSeq RNA Illumina Library v2 construction (nonstranded polyA enrichment) and HiSeq2500 Illumina 2x100bp 2Gb (20M pairend reads) throughput-based sequencing. Transcriptome datasets for both 3D7 and Dd2 were mapped against the 3D7 v3 reference sequence ${ }^{61}$ using HISAT2 2-2.0.0-beta ${ }^{62}$ (-rna-strandness RF -max-intronlen 5000). Read counts were determined using HTSEQ v0.6.0 63 (-r pos $-s$ no). Deconvolution analysis was performed as described previously ${ }^{64}$. Differentially expressed genes were determined using edge $\mathrm{R}^{65}$, with genes expressed at $<=3$ counts per million (cpm) excluded. Because there were no biological replicates for any condition, we used the exact test with dispersion set to 0.01 , as suggested for data concerning genetically identical model organisms

(https://www.bioconductor.org/packages/release/bioc/vignettes/edgeR/inst/doc/edgeRUsersGuide.pdf). We used a false discovery rate (FDR) cut off of 0.01 and a fold change cut off of 2 to call differentially expressed genes.

\section{Phylogenetic analysis}

A dataset of 117 publicly available amino acid sequences from $A B C$ transporters spanning a wide taxonomic range was retrieved with BLASTp (supplementary dataset 2) by querying the Landmark dataset with the pfmrp 1 amino acid sequence (XP_001351050) ${ }^{66}$. These sequences were aligned using MAFFT v. $7.221^{67}$ and used to make phylogenetic reconstructions using IQ-TREE multicore version 1.5.5. The best fitting substitution model was LG+F+R8 as determined by ModelFinder according to $\mathrm{AlC}^{68,69}$. Node support for the inferred maximum likelihood trees was computed using 5000 ultrafast bootstrap replicates ${ }^{68}$.

\section{Declarations}

\section{Acknowledgments}

This work was supported by Portuguese National funds, through the Foundation for Science and Technology (FCT) (projects: UIDB/50026/2020 and UIDP/50026/2020; PTDC/SAU-PAR/28066/2017; 
fellowships: SFRH/BD/129769/2017 to M. S., PD/BD/127826/2016 to C. C., SFRH/BD/145427/2019 to V.P., and contract funding IF/00143/2015/CP1294/CT0001 to P. E. F. and 2020.03113.CEECIND to M.I.V.); by ICVS Scientific Microscopy Platform, member of the national infrastructure PPBI - Portuguese Platform of Bioimaging (PPBI-POCI-01-0145-FEDER-022122); by the project NORTE-01-0145-FEDER028178, supported by Norte Portugal Regional Operational Programme (NORTE 2020), under the PORTUGAL 2020 Partnership Agreement, through the European Regional Development Fund (ERDF); by the ESCMID Research Grant to MIV; and by the NIH (R37 AI050234) to D.A.F.

\section{Author Contributions}

M.S., M.I.V. and P. F. created and designed the study. M.S. constructed strains, performed all in vitro assays and data analysis. C.C. performed susceptibility and cytometry assays. M.T. performed cytometry assays. V.B. performed low folate susceptibility assays. N.S.O. performed in silico phylogeny analysis. A.J.R. and P.F. analysed transcriptomic data. M.S. drafted the manuscript. N.S.O, V.T., P.F., D.A.F., and M.I.V. significantly revised the manuscript. M.I.V. and P.F. supervised the study. All authors reviewed the manuscript and approved the final manuscript.

\section{Declaration of Interests}

The authors declare no competing interests.

\section{References}

1. World Health Organization. World malaria report 2020. WHO Regional Office for Africa 1-232 (2020).

2. Rees, D. C., Johnson, E. \& Lewinson, O. ABC transporters: The power to change. Nat. Rev. Mol. Cell Biol. <background-color:\#FFCC66;bvertical-align:super;>10</background-color:\#FFCC66;bverticalalign:super;>, 218-227 (2009).

3. Ter Beek, J., Guskov, A. \& Slotboom, D. J. Structural diversity of ABC transporters. J. Gen. Physiol. <background-color:\#FFCC66;bvertical-align:super;>143</background-color:\#FFCC66;bverticalalign:super;>, 419-435 (2014).

4. Arnold, F. M. et al. The $A B C$ exporter Irt $A B$ imports and reduces mycobacterial siderophores. Nature <background-color:\#FFCC66;bvertical-align:super;>580</background-color:\#FFCC66;bverticalalign:super;>, 413-417 (2020).

5. Lee, M. et al. The ABC transporter AtABCB14 is a malate importer and modulates stomatal response to CO2. Nat. Cell Biol. <background-color:\#FFCC66;bvertical-align:super;>10</backgroundcolor:\#FFCC66;bvertical-align:super;>, 1217-1223 (2008).

6. Lewinson, O. \& Livnat-Levanon, N. Mechanism of Action of ABC Importers: Conservation, Divergence, and Physiological Adaptations. J. Mol. Biol. <background-color:\#FFCC66;bverticalalign:super;>429</background-color:\#FFCC66;bvertical-align:super;>, 606-619 (2017). 
7. Rempel, S. et al. A mycobacterial $A B C$ transporter mediates the uptake of hydrophilic compounds. Nature<background-color:\#FFCC66;bvertical-align:super;>580</background-color:\#FFCC66;bverticalalign:super;>, 409-412 (2020).

8. Shitan, N. et al. Involvement of CjMDR1, a plant multidrug-resistance-type ATP-binding cassette protein, in alkaloid transport in Coptis japonica. Proc. Natl. Acad. Sci. U. S. A. <bverticalalign:super;>100</bvertical-align:super;>, 751-756 (2003).

9. Terasaka, K. et al. PGP4, an ATP binding cassette P-glycoprotein, catalyzes auxin transport in Arabidopsis thaliana roots. Plant Cell <background-color:\#FFCC66;bverticalalign:super;>17</background-color:\#FFCC66;bvertical-align:super;>, 2922-2939 (2005).

10. Wang, Z., Hu, W. \& Zheng, H. Pathogenic siderophore ABC importer YbtPQ adopts a surprising fold of exporter. Sci. Adv. <background-color:\#FFCC66;bvertical-align:super;>6</backgroundcolor:\#FFCC66;bvertical-align:super;>, eaay7997 (2020).

11. Wanke, D. \& Üner Kolukisaoglu, H. An update on the ABCC transporter family in plants: Many genes, many proteins, but how many functions? Plant Biol. <background-color:\#FFCC66;bverticalalign:super;>12</background-color:\#FFCC66;bvertical-align:super;>, 15-25 (2010).

12. Thomas, C. et al. Structural and functional diversity calls for a new classification of $A B C$ transporters. FEBS Lett. <background-color:\#FFCC66;bvertical-align:super;>594</backgroundcolor:\#FFCC66;bvertical-align:super;>, 3767-3775 (2020).

13. Kavishe, R. A. et al. Localization of the ATP-binding cassette (ABC) transport proteins PfMRP1, PfMRP2, and PfMDR5 at the Plasmodium falciparum plasma membrane. Malar. J. <backgroundcolor:\#FFCC66;bvertical-align:super;>8</background-color:\#FFCC66;bvertical-align:super;>, 205 (2009).

14. Veiga, M. I. et al. Globally prevalent PfMDR1 mutations modulate Plasmodium falciparum susceptibility to artemisinin-based combination therapies. Nat. Commun. <backgroundcolor:\#FFCC66;bvertical-align:super;>7</background-color:\#FFCC66;bvertical-align:super;>, 11553 (2016).

15. Duraisingh, M. T. \& Cowman, A. F. Contribution of the $p f m d r 1$ gene to antimalarial drug-resistance. Acta Trop. <background-color:\#FFCC66;bvertical-align:super;>94</backgroundcolor:\#FFCC66;bvertical-align:super;>, 181-190 (2005).

16. Dahlström, S. et al. Plasmodium falciparum multidrug resistance protein 1 and artemisinin-based combination therapy in Africa. J. Infect. Dis. <background-color:\#FFCC66;bverticalalign:super;>200</background-color:\#FFCC66;bvertical-align:super;>, 1456-1464 (2009).

17. Dahlström, S., Veiga, M. I., Mårtensson, A., Björkman, A. \& Gil, J. P. Polymorphism in Pfmrp1 (Plasmodium falciparum multidrug resistance protein 1) amino acid 1466 associated with resistance to sulfadoxine-pyrimethamine treatment. Antimicrob. Agents Chemother. <backgroundcolor:\#FFCC66;bvertical-align:super;>53</background-color:\#FFCC66;bvertical-align:super;>, 25532556 (2009). 
18. Mok, S. et al. Structural polymorphism in the promoter of pfmrp2 confers Plasmodium falciparum tolerance to quinoline drugs. Mol. Microbiol. <background-color:\#FFCC66;bverticalalign:super;>91</background-color:\#FFCC66;bvertical-align:super;>, 918-934 (2014).

19. Nogueira, F., Lopes, D., Alves, A. C. \& Estólio Do Rosário, V. Plasmodium falciparum multidrug resistance protein (MRP) gene expression under chloroquine and mefloquine challenge. J. Cell Anim. Biol. <background-color:\#FFCC66;bvertical-align:super;>2</background-color:\#FFCC66;bverticalalign:super;>, 10-20 (2008).

20. Raj, D. K. et al. Disruption of a Plasmodium falciparum multidrug resistance-associated protein (PfMRP) alters its fitness and transport of antimalarial drugs and glutathione. J. Biol. Chem. <background-color:\#FFCC66;bvertical-align:super;>284</background-color:\#FFCC66;bverticalalign:super;>, 7687-7696 (2009).

21. Veiga, M. I. et al. Complex polymorphisms in the Plasmodium falciparum multidrug resistance protein 2 gene and its contribution to antimalarial response. Antimicrob. Agents Chemother. <background-color:\#FFCC66;bvertical-align:super;>58</background-color:\#FFCC66;bverticalalign:super;>, 7390-7397 (2014).

22. Veiga, M. I. et al. Novel polymorphisms in Plasmodium falciparum ABC transporter genes are associated with major ACT antimalarial drug resistance. PloS One <backgroundcolor:\#FFCC66;bvertical-align:super;>6</background-color:\#FFCC66;bvertical-align:super;>, e20212 (2011).

23. Atamna, H. \& Ginsburg, H. The malaria parasite supplies glutathione to its host cell - Investigation of glutathione transport and metabolism in human erythrocytes infected with Plasmodium falciparum. Eur. J. Biochem. <background-color:\#FFCC66;bvertical-align:super;>250</backgroundcolor:\#FFCC66;bvertical-align:super;>, 670-679 (1997).

24. Bozdech, Z. \& Ginsburg, H. Antioxidant defense in Plasmodium falciparum - data mining of the transcriptome. Malar. J. <background-color:\#FFCC66;bvertical-align:super;>3</backgroundcolor:\#FFCC66;bvertical-align:super;>, 23 (2004).

25. Rijpma, S. R. et al. MRP1 mediates folate transport and antifolate sensitivity in Plasmodium falciparum. FEBS Lett. <background-color:\#FFCC66;bvertical-align:super;>590</backgroundcolor:\#FFCC66;bvertical-align:super;>, 482-492 (2016).

26. $\mathrm{Mu}, \mathrm{J}$. et al. Multiple transporters associated with malaria parasite responses to chloroquine and quinine. Mol. Microbiol. <background-color:\#FFCC66;bvertical-align:super;>49</backgroundcolor:\#FFCC66;bvertical-align:super;>, 977-989 (2003).

27. Phompradit, P., Muhamad, P., Chaijaroenkul, W. \& Na-Bangchang, K. Genetic polymorphisms of candidate markers and in vitro susceptibility of Plasmodium falciparum isolates from Thai-Myanmar border in relation to clinical response to artesunate-mefloquine combination. Acta Trop. <background-color:\#FFCC66;bvertical-align:super;>139</background-color:\#FFCC66;bverticalalign:super;>, 77-83 (2014). 
28. Phompradit, P., Muhamad, P., Wisedpanichkij, R., Chaijaroenkul, W. \& Na-Bangchang, K. Four years' monitoring of in vitro sensitivity and candidate molecular markers of resistance of Plasmodium falciparum to artesunate-mefloquine combination in the Thai-Myanmar border. Malar. J. <background-color:\#FFCC66;bvertical-align:super;>13</background-color:\#FFCC66;bverticalalign:super;>, 23 (2014).

29. Rijpma, S. R. et al. Multidrug ATP-binding cassette transporters are essential for hepatic development of Plasmodium sporozoites. Cell. Microbiol. <background-color:\#FFCC66;bverticalalign:super;>18</background-color:\#FFCC66;bvertical-align:super;>, 369-383 (2016).

30. Antony, H. A., Pathak, V., Parija, S. C., Ghosh, K. \& Bhattacherjee, A. Transcriptomic Analysis of Chloroquine-Sensitive and Chloroquine-Resistant Strains of Plasmodium falciparum: Toward Malaria Diagnostics and Therapeutics for Global Health. OMICS J. Integr. Biol. <backgroundcolor:\#FFCC66;bvertical-align:super;>20</background-color:\#FFCC66;bvertical-align:super;>, 424432 (2016).

31. Guinet, F. et al. A developmental defect in Plasmodium falciparum male gametogenesis. J. Cell Biol. <background-color:\#FFCC66;bvertical-align:super;>135</background-color:\#FFCC66;bverticalalign:super;>, 269-278 (1996).

32. Walliker, D. et al. Genetic analysis of the human malaria parasite Plasmodium falciparum. Science <background-color:\#FFCC66;bvertical-align:super;>236</background-color:\#FFCC66;bverticalalign:super;>, 1661-1666 (1987).

33. Ahouidi, A. et al. An open dataset of Plasmodium falciparum genome variation in 7,000 worldwide samples. Wellcome Open Res. <background-color:\#FFCC66;bvertical-align:super;>6</backgroundcolor:\#FFCC66;bvertical-align:super;>, 42 (2021).

34. Johnson, D. J. et al. Evidence for a central role for PfCRT in conferring Plasmodium falciparum resistance to diverse antimalarial agents. Mol. Cell<background-color:\#FFCC66;bverticalalign:super;>15</background-color:\#FFCC66;bvertical-align:super;>, 867-877 (2004).

35. Kim, J. et al. Structure and drug resistance of the Plasmodium falciparum transporter PfCRT. Nature <background-color:\#FFCC66;bvertical-align:super;>576</background-color:\#FFCC66;bverticalalign:super;>, 315-320 (2019).

36. Ross, L. S. et al. Emerging Southeast Asian PfCRT mutations confer Plasmodium falciparum resistance to the first-line antimalarial piperaquine. Nat. Commun. <backgroundcolor:\#FFCC66;bvertical-align:super;>9</background-color:\#FFCC66;bvertical-align:super;>, 3314 (2018).

37. Fidock, D. A., Nomura, T. \& Wellems, T. E. Cycloguanil and Its Parent Compound Proguanil Demonstrate Distinct Activities against Plasmodium falciparum Malaria Parasites Transformed with Human Dihydrofolate Reductase. Mol. Pharmacol. <background-color:\#FFCC66;bverticalalign:super;>54</background-color:\#FFCC66;bvertical-align:super;>, 1140-1147 (1998).

38. Wang, P., Read, M., Sims, P. F. G. \& Hyde, J. E. Sulfadoxine resistance in the human malaria parasite Plasmodium falciparum is determined by mutations in dihydropteroate synthetase and an additional 
factor associated with folate utilization. Mol. Microbiol. <background-color:\#FFCC66;bverticalalign:super;>23</background-color:\#FFCC66;bvertical-align:super;>, 979-986 (1997).

39. da Costa, K. M. et al. Functional Characterization of ABCC Proteins from Trypanosoma cruzi and Their Involvement with Thiol Transport. Front. Microbiol. <background-color:\#FFCC66;bverticalalign:super;>9</background-color:\#FFCC66;bvertical-align:super;>, 205 (2018).

40. Gekeler, V., Ise, W., Sanders, K. H., Ulrich, W. R. \& Beck, J. The leukotriene LTD4 receptor antagonist MK571 specifically modulates MRP associated multidrug resistance. Biochem. Biophys. Res. Commun. <background-color:\#FFCC66;bvertical-align:super;>208</backgroundcolor:\#FFCC66;bvertical-align:super;>, 345-352 (1995).

41. Tivnan, A. et al. Inhibition of multidrug resistance protein 1 (MRP1) improves chemotherapy drug response in primary and recurrent glioblastoma multiforme. Front. Neurosci. <backgroundcolor:\#FFCC66;bvertical-align:super;>9</background-color:\#FFCC66;bvertical-align:super;>, 218 (2015).

42. Shafik, S. H. et al. The natural function of the malaria parasite's chloroquine resistance transporter. Nat. Commun. <background-color:\#FFCC66;bvertical-align:super;>11</backgroundcolor:\#FFCC66;bvertical-align:super;>, 3922 (2020).

43. Calçada, C. et al. Expansion of a Specific Plasmodium falciparum PfMDR1 Haplotype in Southeast Asia with Increased Substrate Transport. mBio <background-color:\#FFCC66;bverticalalign:super;>11</background-color:\#FFCC66;bvertical-align:super;>, e02093-20 (2020).

44. Friedrich, O., Reiling, S., Wunderlich, J. \& Rohrbach, P. Assessment of Plasmodium falciparum PfMDR1 transport rates using Fluo-4. J. Cell. Mol. Med. <background-color:\#FFCC66;bverticalalign:super;>18</background-color:\#FFCC66;bvertical-align:super;>, 1851-1862 (2014).

45. World Health Organization. Serum and Red Blood Cell Folate Concentrations for Assessing Folate Status in Populations. Vitam. Miner. Nutr. Inf. Syst. 1-7 (2015).

46. Wang, P., Brobey, R. K., Horii, T., Sims, P. F. \& Hyde, J. E. Utilization of exogenous folate in the human malaria parasite Plasmodium falciparum and its critical role in antifolate drug synergy. Mol. Microbiol. <background-color:\#FFCC66;bvertical-align:super;>32</backgroundcolor:\#FFCC66;bvertical-align:super;>, 1254-1262 (1999).

47. Ursing, J., Zakeri, S., Gil, J. P. \& Björkman, A. Quinoline resistance associated polymorphisms in the pfcrt, pfmdr1 and pfmrp genes of Plasmodium falciparum in Iran. Acta Trop. <backgroundcolor:\#FFCC66;bvertical-align:super;>97</background-color:\#FFCC66;bvertical-align:super;>, 352356 (2006).

48. Chen, Z. S. et al. Analysis of methotrexate and folate transport by multidrug resistance protein 4 (ABCC4): MRP4 is a component of the methotrexate efflux system. Cancer Res. <backgroundcolor:\#FFCC66;bvertical-align:super;>62</background-color:\#FFCC66;bvertical-align:super;>, 31443150 (2002).

49. Hooijberg, J. H. et al. Antifolate resistance mediated by the multidrug resistance proteins MRP1 and MRP2. Cancer Res. <background-color:\#FFCC66;bvertical-align:super;>59</background- 
color:\#FFCC66;bvertical-align:super;>, 2532-2535 (1999).

50. Zeng, H., Chen, Z. S., Belinsky, M. G., Rea, P. A. \& Kruh, G. D. Transport of methotrexate (MTX) and folates by multidrug resistance protein (MRP) 3 and MRP1: Effect of polyglutamylation on MTX transport. Cancer Res. <background-color:\#FFCC66;bvertical-align:super;>61</backgroundcolor:\#FFCC66;bvertical-align:super;>, 7225-7232 (2001).

51. Salcedo-Sora, J. E. et al. The molecular basis of folate salvage in plasmodium falciparum: Characterization of two folate transporters. J. Biol. Chem. <background-color:\#FFCC66;bverticalalign:super;>286</background-color:\#FFCC66;bvertical-align:super;>, 44659-44668 (2011).

52. Wang, P., Wang, Q., Sims, P. F. G. \& Hyde, J. E. Characterisation of exogenous folate transport in Plasmodium falciparum. Mol. Biochem. Parasitol. <background-color:\#FFCC66;bverticalalign:super;>154</background-color:\#FFCC66;bvertical-align:super;>, 40-51 (2007).

53. Ward, A., Reyes, C. L., Yu, J., Roth, C. B. \& Chang, G. Flexibility in the ABC transporter MsbA: Alternating access with a twist. Proc. Natl. Acad. Sci. U. S. A. <bvertical-align:super;>104</bverticalalign:super;>, 19005-19010 (2007).

54. Djuika, C. F. et al. Prokaryotic ancestry and gene fusion of a dual localized peroxiredoxin in malaria parasites. Microb. Cell<background-color:\#FFCC66;bvertical-align:super;>2</backgroundcolor:\#FFCC66;bvertical-align:super;>, 5-13 (2015).

55. Mu, J. et al. Chromosome-wide SNPs reveal an ancient origin for Plasmodium falciparum. Nature <background-color:\#FFCC66;bvertical-align:super;>418</background-color:\#FFCC66;bverticalalign:super;>, 323-326 (2002).

56. Huang, J., Mullapudi, N., Sicheritz-Ponten, T. \& Kissinger, J. C. A first glimpse into the pattern and scale of gene transfer in the Apicomplexa. Int. J. Parasitol. <background-color:\#FFCC66;bverticalalign:super;>34</background-color:\#FFCC66;bvertical-align:super;>, 265-274 (2004).

57. Huang, J. et al. Phylogenomic evidence supports past endosymbiosis, intracellular and horizontal gene transfer in Cryptosporidium parvum. Genome Biol. <background-color:\#FFCC66;bverticalalign:super;>5</background-color:\#FFCC66;bvertical-align:super;>, R88 (2004).

58. Striepen, B. et al. Gene transfer in the evolution of parasite nucleotide biosynthesis. Proc. Natl. Acad. Sci. U. S. A. <bvertical-align:super;>101</bvertical-align:super;>, 3154-3159 (2004).

59. Gardner, M. J. et al. Genome sequence of the human malaria parasite Plasmodium falciparum. Nature<background-color:\#FFCC66;bvertical-align:super;>419</background-color:\#FFCC66;bverticalalign:super;>, 498-511 (2002).

60. Amaratunga, C., Neal, A. T. \& Fairhurst, R. M. Flow cytometry-based analysis of artemisinin-resistant Plasmodium falciparum in the ring-stage survival assay. Antimicrob. Agents Chemother. <background-color:\#FFCC66;bvertical-align:super;>58</background-color:\#FFCC66;bverticalalign:super;>, 4938-4940 (2014).

61. Böhme, U., Otto, T. D., Sanders, M., Newbold, C. I. \& Berriman, M. Progression of the canonical reference malaria parasite genome from 2002-2019. Wellcome Open Res. <background- 
color:\#FFCC66;bvertical-align:super;>4</background-color:\#FFCC66;bvertical-align:super;>, 58 (2019).

62. Kim, D., Paggi, J. M., Park, C., Bennett, C. \& Salzberg, S. L. Graph-based genome alignment and genotyping with HISAT2 and HISAT-genotype. Nat. Biotechnol. <background-color:\#FFCC66;bverticalalign:super;>37</background-color:\#FFCC66;bvertical-align:super;>, 907-915 (2019).

63. Anders, S., Pyl, P. T. \& Huber, W. HTSeq-a Python framework to work with high-throughput sequencing data. Bioinforma. Oxf. Engl. <background-color:\#FFCC66;bverticalalign:super;>31</background-color:\#FFCC66;bvertical-align:super;>, 166-169 (2015).

64. Aunin, E. et al. Genomic and transcriptomic evidence for descent from Plasmodium and loss of blood schizogony in Hepatocystis parasites from naturally infected red colobus monkeys. PLoS Pathog. <background-color:\#FFCC66;bvertical-align:super;>16</background-color:\#FFCC66;bverticalalign:super;>, e1008717 (2020).

65. Robinson, M. D., McCarthy, D. J. \& Smyth, G. K. edgeR: a Bioconductor package for differential expression analysis of digital gene expression data. Bioinforma. Oxf. Engl. <backgroundcolor:\#FFCC66;bvertical-align:super;>26</background-color:\#FFCC66;bvertical-align:super;>, 139140 (2010).

66. McGinnis, S. \& Madden, T. L. BLAST: at the core of a powerful and diverse set of sequence analysis tools. Nucleic Acids Res. <background-color:\#FFCC66;bvertical-align:super;>32</backgroundcolor:\#FFCC66;bvertical-align:super;>, W20-25 (2004).

67. Katoh, K. \& Standley, D. M. MAFFT Multiple Sequence Alignment Software Version 7: Improvements in Performance and Usability. Mol. Biol. Evol. <background-color:\#FFCC66;bverticalalign:super;>30</background-color:\#FFCC66;bvertical-align:super;>, 772-780 (2013).

68. Minh, B. Q., Nguyen, M. A. T. \& von Haeseler, A. Ultrafast Approximation for Phylogenetic Bootstrap. Mol. Biol. Evol. <background-color:\#FFCC66;bvertical-align:super;>30</backgroundcolor:\#FFCC66;bvertical-align:super;>, 1188-1195 (2013).

69. Nguyen, L.-T., Schmidt, H. A., von Haeseler, A. \& Minh, B. Q. IQ-TREE: A Fast and Effective Stochastic Algorithm for Estimating Maximum-Likelihood Phylogenies. Mol. Biol. Evol. <backgroundcolor:\#FFCC66;bvertical-align:super;>32</background-color:\#FFCC66;bvertical-align:super;>, 268274 (2015).

\section{Figures}


a

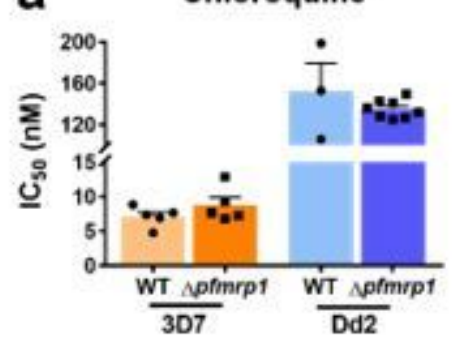

Dihydroartemisinin

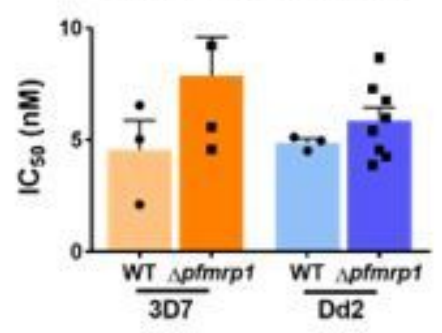

WR99210

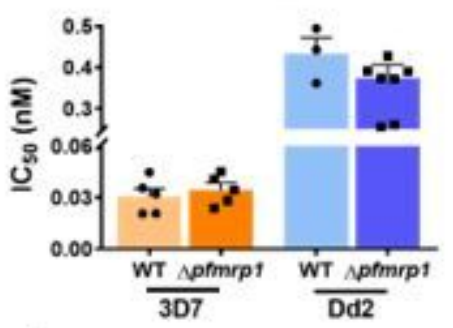

b Folate
Mefloquine

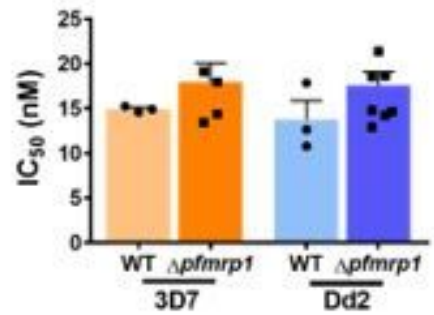

Pyrimethamine

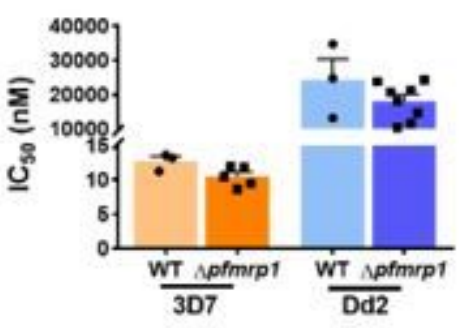

Methotrexate

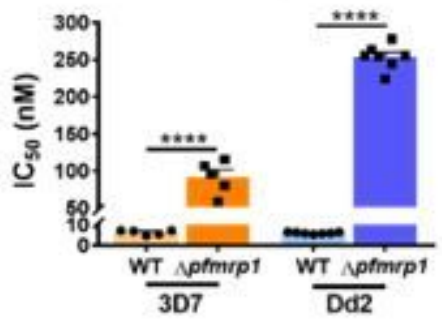

Methotrexate
Lumefantrine

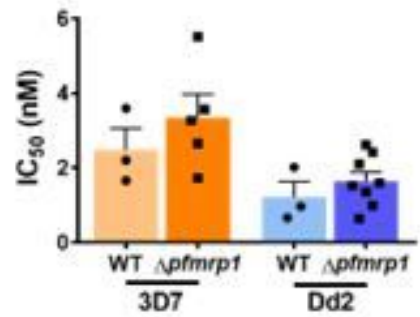

Trimethoprim

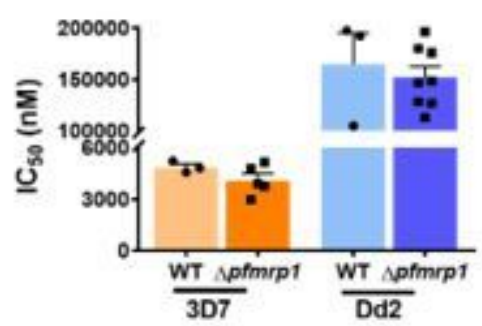

Aminopterin

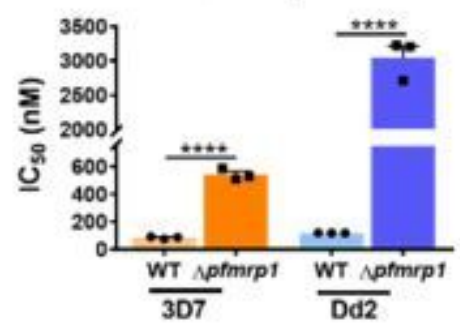

Aminopterin<smiles>CN(Cc1cnc2c(n1)[C@]([NH3+])(Cc1ccccc1)NC(N)=N2)c1ccc(C(=O)NC(CCC(=O)O)C(=O)O)cc1</smiles>

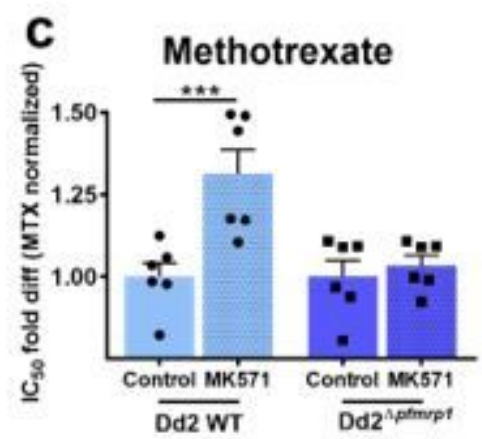

d Methotrexate

Aminopterin
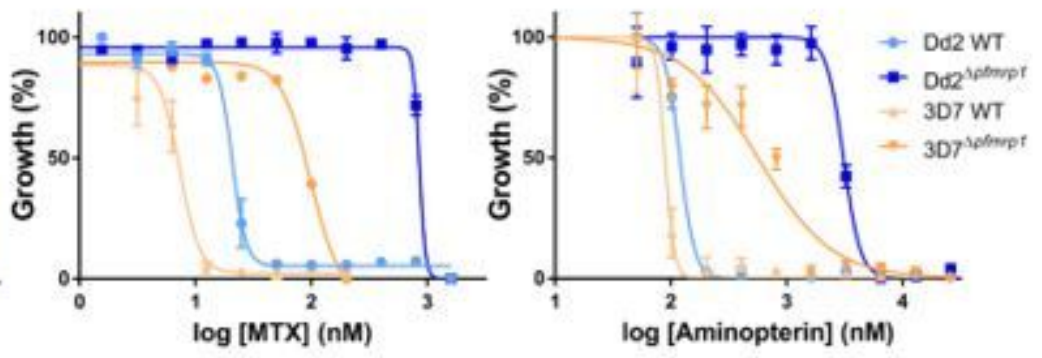

Figure 1

pfmrp1 disruption leads to decreased susceptibility to folate analogs.

a - In vitro $\mathrm{IC}_{50}$ antimalarial responses for $\Delta$ pfmrp1 and wild-type (WT) $3 \mathrm{D} 7$ and $\mathrm{Dd} 2$ strains. Values of $I_{50}$ are presented as the mean \pm SEM for chloroquine (CQ), mefloquine (MQ), lumefantrine (LUM), dihydroartemisinin (DHA), pyrimethamine (PYR), trimethoprim (TMP), WR99210 (WR), methotrexate 
(MTX) and aminopterin (AMT). Statistically significant differences were observed for MTX and AMT,

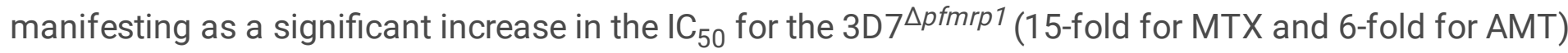
and Dd2 ${ }^{\triangle p f m r p 1}$ (40-fold for MTX and 25-fold for AMT) strains relative to their respective parental control strains. Assays were performed in at least 3 independent replicates (black squares and triangles). Statistical evaluations comparing WT and $\Delta p f m r p 1$ strains were performed using two-tailed unpaired ttests. $* \star \star * P<0.0001$.

b - The PfDHFR inhibitors MTX and AMT are folate analogs. Red circles represent functional group differences between analogs and folate.

c - In vitro $\mathrm{IC}_{50}$ responses for Dd2 WT and Dd2 ${ }^{\Delta p f m r p 1}$ strains in the presence of only MTX (Control) or MTX plus MK571, a PfMRP1 chemical inhibitor, normalized against control conditions. $\mathrm{IC}_{50}$ values are presented as the mean \pm SEM. PFMRP1 chemical inhibition led to a significant decrease in MTX susceptibility only for the Dd2 WT strain. Assays were performed in 6 independent assays (black squares and triangles). Statistical evaluation comparing MTX only (Control) and MTX plus MK571 conditions was performed using two-tailed unpaired t-tests. ${ }^{*} * \mathrm{P}<0.001$.

$\mathbf{d}-$ In vitro growth response curves for MTX and AMT of Dd2 and 3D7 WT and $\Delta p f m r p 1$ strains. Significant increases in the $I C_{50}$ values for the $D d 2^{\triangle p f m r p 1}$ and $3 D 7^{\triangle p f m r p 1}$ were observed for both folate analogs. Assays were performed in at least 3 independent replicates. 
a

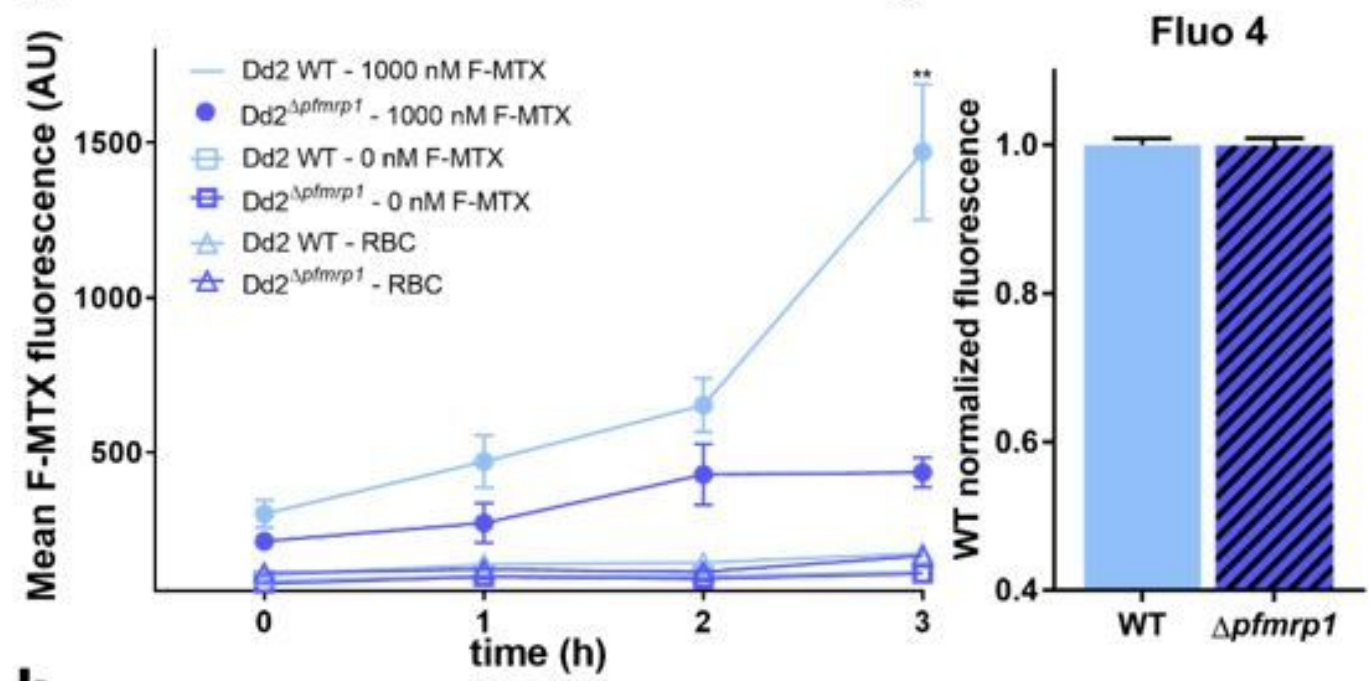

b

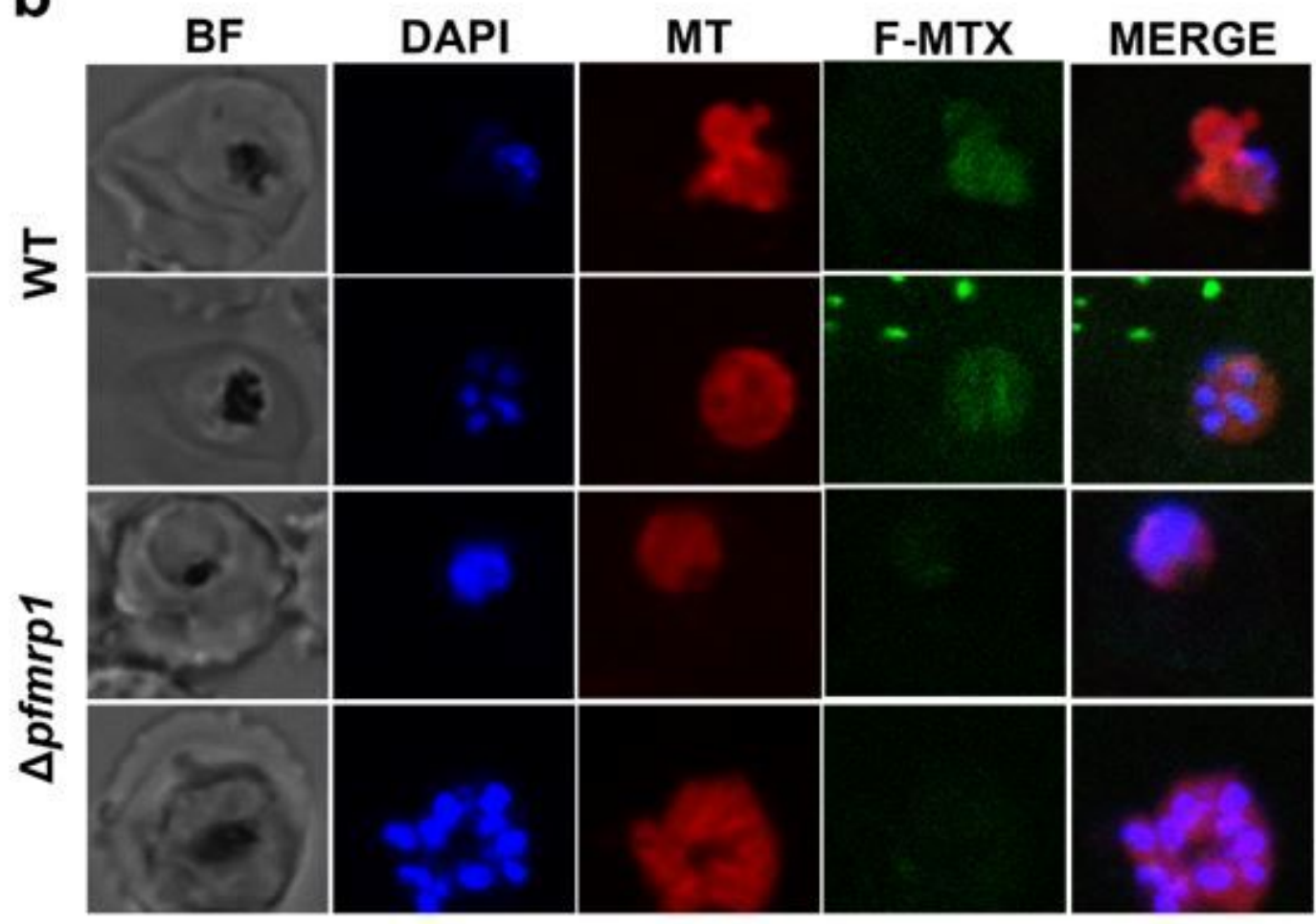

F-MTX
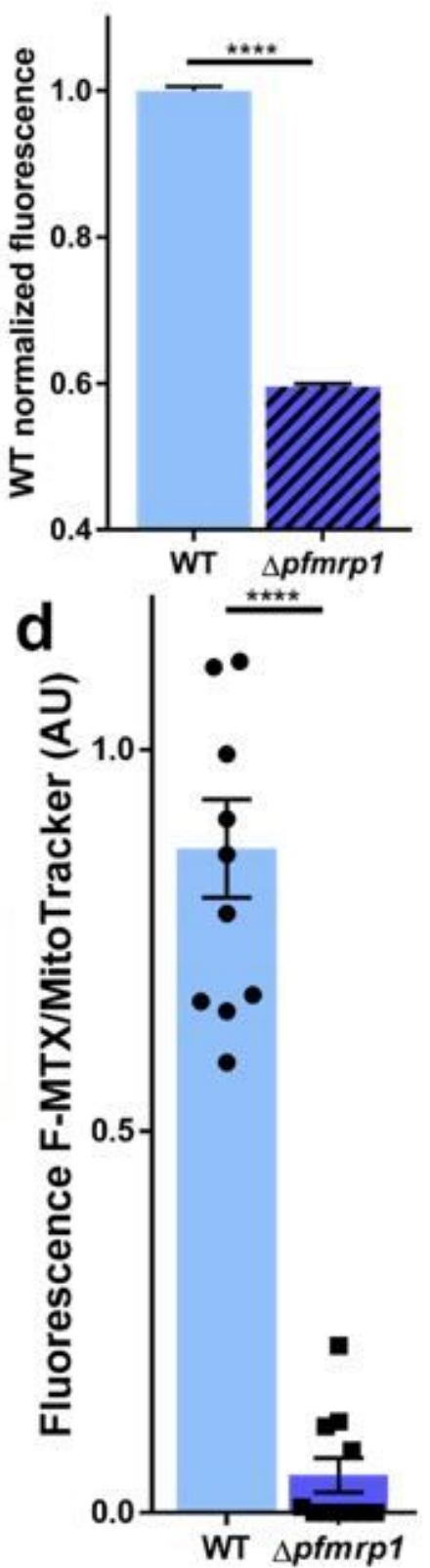

Figure 2

pfmrp1-disrupted parasites accumulate less fluorescein methotrexate (F-MTX) than WT parasites.

a - Flow cytometry-based F-MTX accumulation assay over time. F-MTX was used at $1000 \mathrm{nM} .0 \mathrm{nM}$ of FMTX and uninfected RBCs were used as controls for baseline and autofluorescence. $\Delta p f m r p 1$ parasites accumulated less F-MTX than WT, a trend that was maintained over 3 hours at the end of which WT parasites $(1470 \pm 219 \mathrm{AU})$ had $\approx 3$-fold more F-MTX fluorescence compared to $\Delta$ pfmrp 1 parasites $(434 \pm 49$ $A U)$. Assays were performed in 2 independent replicates with 3 technical replicates. AU - Arbitrary 
fluorescence units. Mean \pm SEM values are presented. Statistical evaluations comparing WT and $\Delta p f m r p 1$ strains were performed using two-tailed unpaired t-tests. ${ }^{\star *} \mathrm{P}<0.01$.

b - Quantification from the cytometry-based F-MTX accumulation assay normalized to WT fluorescence after 3 hours of incubation. Quantification with Fluo-4 (that enters the parasite cytoplasm through passive diffusion) was used as a control for non-specific transport. Fluo-4 was incorporated to the same degree in WT and $\Delta$ ffmrp 1 parasites as expected, while F-MTX was $\approx 1.7$-fold more fluorescent in WT parasites. Statistical evaluation were performed using two-tailed unpaired t-test. ${ }^{\star} * \star * P<0.0001$.

c - Confocal microscopy-based MTX accumulation assay after 3 hours of F-MTX incubation. BF - Bright field; DAPI - 4',6-diamidino-2-phenylindole; MT - MitoTracker. MitoTracker was used to stain live parasites. $\Delta$ ffmrp 1 parasites exhibited less fluorescence than WT (representative parasites). The image was cropped around a single parasite, and the different channels were merged using FlowJ software.

d - Quantification from the microscopy-based F-MTX accumulation assay, on panel C, as a ratio of FMTX/MitoTracker fluorescence. 10 parasites were counted for each condition. There was a significant difference of the fluorescence ratio between WT and $\Delta p f m r p 1$ strains. AU - Arbitrary units. Statistical evaluations were performed using two-tailed unpaired t-tests. ${ }^{*} \star \star * P<0.0001$. 

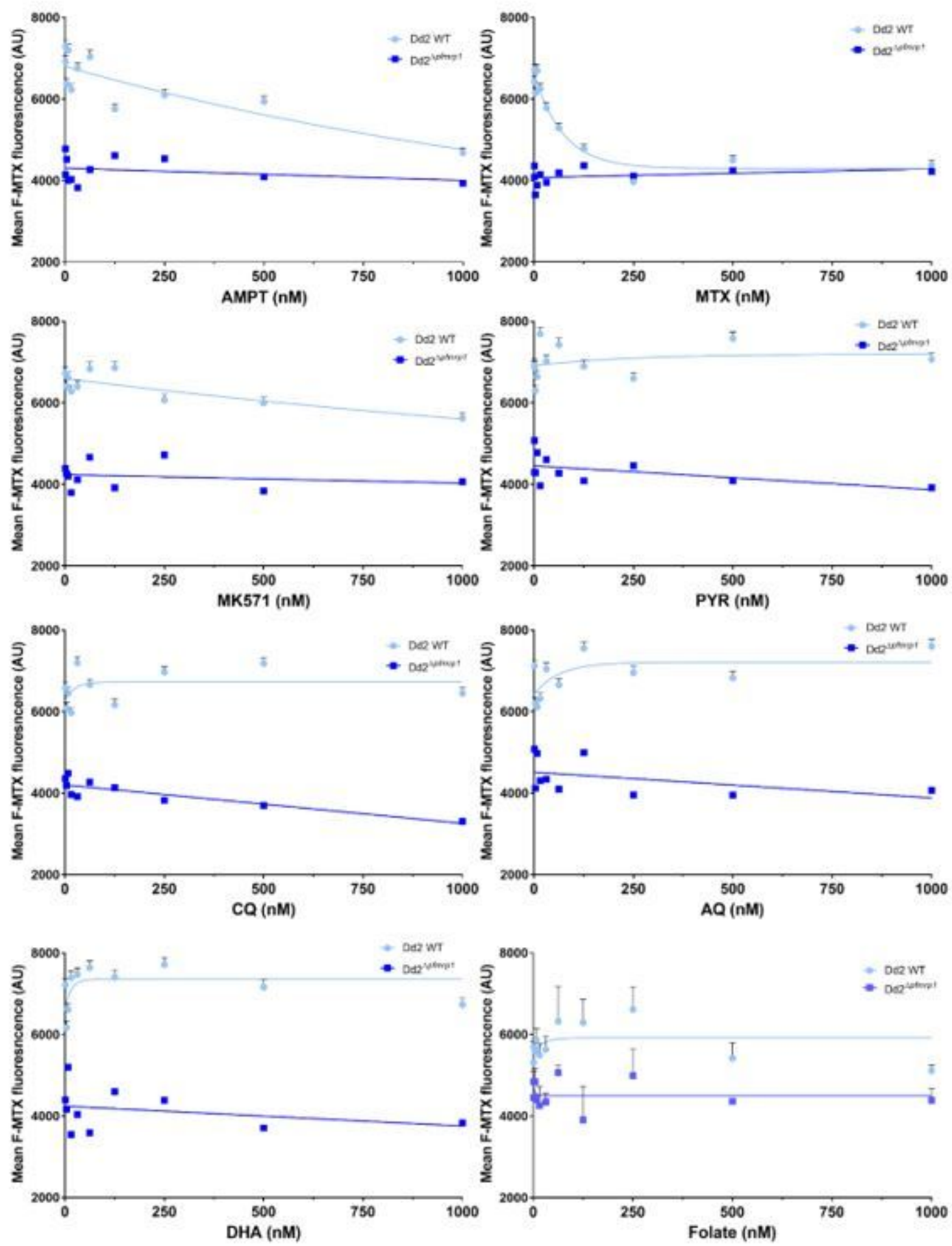

Figure 3

Folate analogs compete with fluorescein methotrexate (F-MTX) for PfMRP1 transport.

Flow cytometry competition assays using a range of compounds concentrations from 0 to $1000 \mathrm{nM}$ with a fixed concentration of 1000 nM F-MTX. Incubations proceeded for 3 hours prior to detection of F-MTX. Aminopterin (AMT), methotrexate (MTX) and MK571 showed decreasing levels of F-MTX accumulation 
with increased drug concentrations in the Dd2 WT strain, an effect that was absent in the Dd2 ${ }^{\triangle p f m r p} 1$ line. No effects were observed for chloroquine (CQ), amodiaquine (AQ), dihydroartemisinin (DHA) and folate. Assays were performed in 2 independent replicates with 3 technical replicates. One independent replicate is presented due to inherent variations in absolute fluorescence. The other replicate presented identical results for all compounds. Mean $\pm S E M$ values are presented.
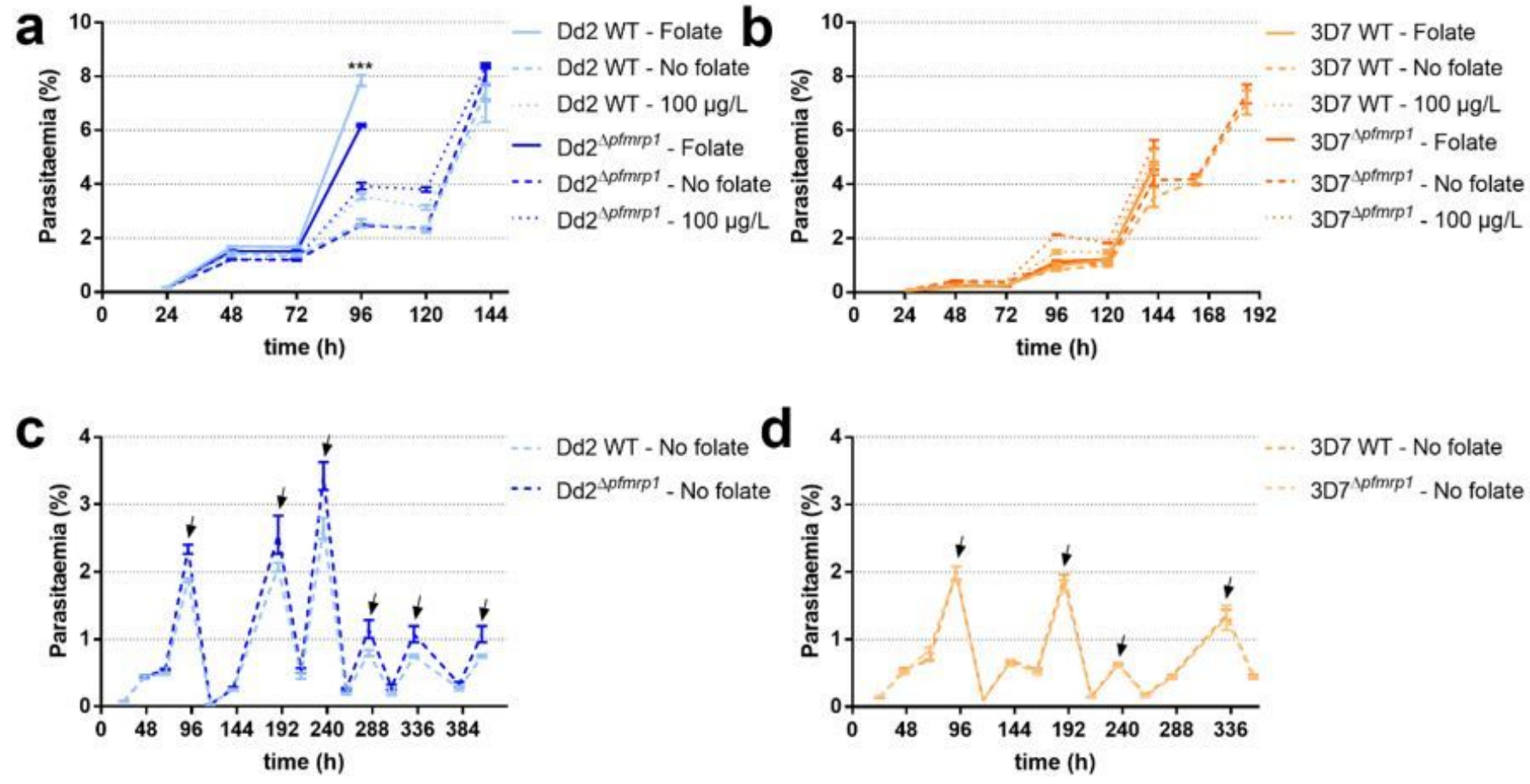

\section{Figure 4}

\section{Impact of folate on growth of wild-type (WT) and $\Delta p f m r p 1$ lines.}

$\mathbf{a} / \mathbf{b}$ - Parasite growth monitored along time in RPMI-based medium with regular concentration of folate (1 mg/L), $100 \mu \mathrm{g} / \mathrm{L}$ folate, or no folate for Dd2 (a) and 3D7 (a) strains after growth for two weeks under folate starvation. $D d 2^{\triangle p f m r p 1}$ showed a significant impairment of growth compared to Dd2 WT under regular conditions, an effect that was not detectable in 3D7 parasites. Dd2 propagated more slowly in medium with reduced concentration of folate compared to regular medium, while 3D7 growth was independent of folate. These data need careful interpretation as the RBCs used for parasite culture naturally contain folate. Mean $\pm S E M$ values are presented. Assays were performed in 3 independent replicates. ${ }^{* \star *} \mathrm{P}<0.001$.

c/d - Parasite growth was monitored over time in RPMI medium without folic acid supplementation. After parasites grew to near $2 \%$ parasitemia they were diluted multiple times to eliminate confounding effects regarding initial parasitemia and folate pools. There was no difference in growth between WT and 

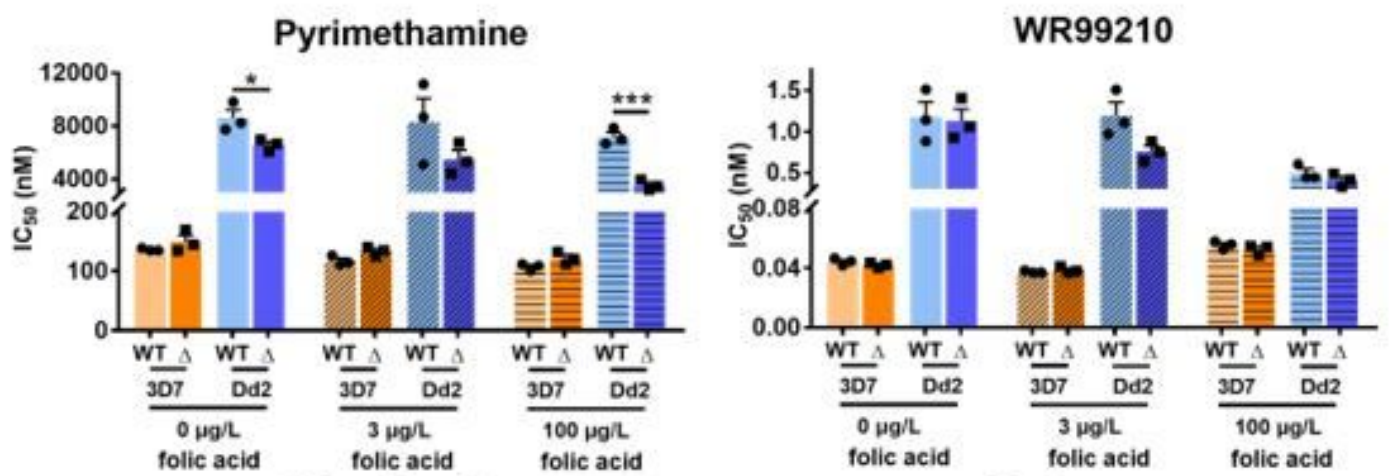

\section{Trimethoprim}
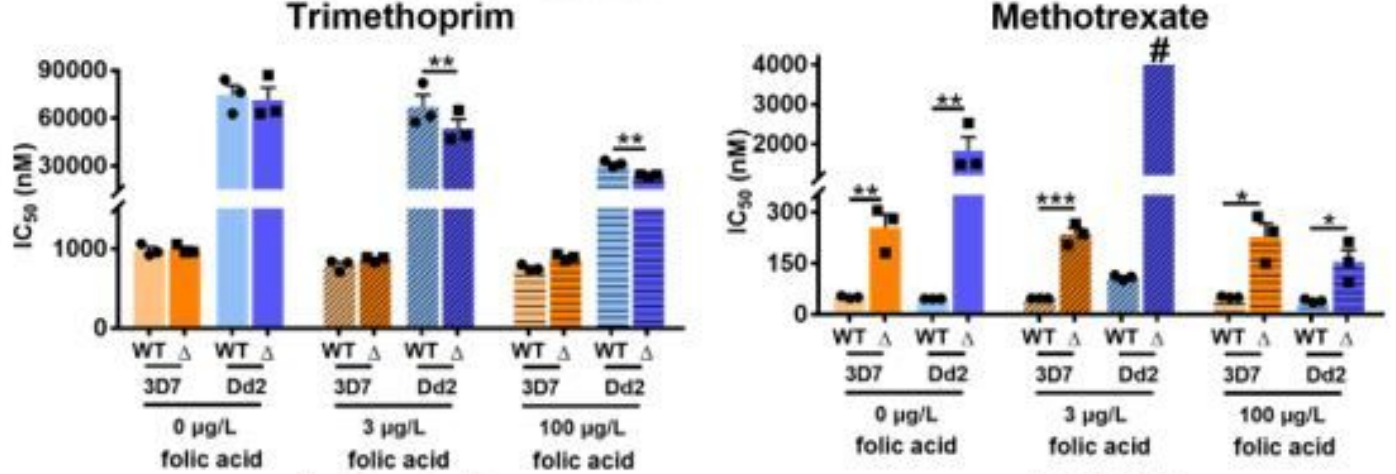

Aminopterin

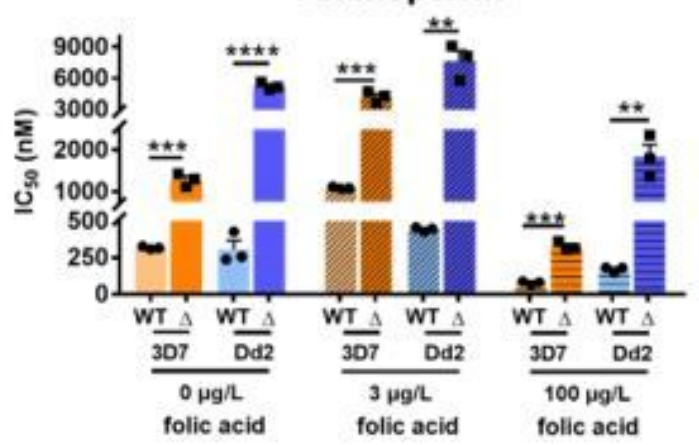

\section{DSM265}

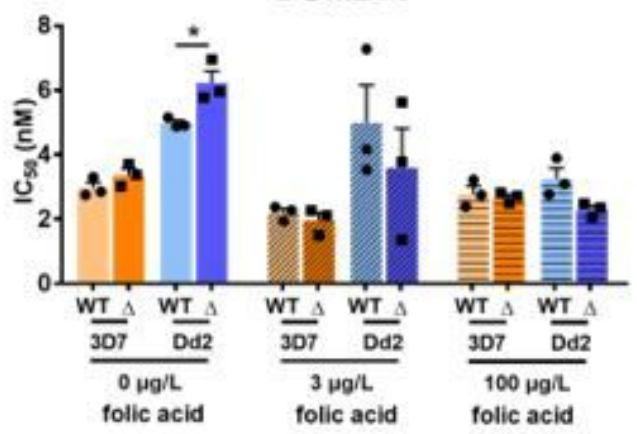

Ferroquine

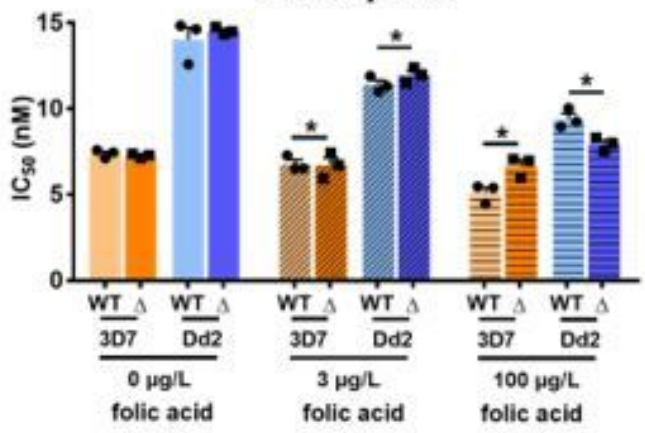

Figure 5

Folic acid impacts parasite drug responses 
In vitro $\mathrm{IC}_{50}$ responses for $\Delta p f m r p 1$ and wild-type (WT) $3 \mathrm{D} 7$ and $\mathrm{Dd} 2$ strains, with varying concentrations of exogenous folate $\left(100 \mu \mathrm{g} / \mathrm{L}, 3 \mu \mathrm{g} / \mathrm{L}\right.$ and no exogenous folate). $\mathrm{IC}_{50}$ values are presented as mean \pm SEM for pyrimethamine (PYR), trimethoprim (TMP), WR99210 (WR), aminopterin (AMT), methotrexate (MTX), ferroquine and DSM265. All compounds are antifolates, except for ferroquine and DSM265, which are functionally different. \#: bar represents a higher $\mathrm{IC}_{50}$ value than that indicated due to a greatly reduced susceptibility to compound that was beyond the limit of detection in this assay. Mean $\pm S E M$ values are presented. Assays were performed in 3 independent replicates. Statistical evaluations comparing WT and $\triangle p f m r p 1$ strains were performed using two-tailed unpaired t-tests. ${ }^{\star} \mathrm{P}<0.05 ;{ }^{* \star P}<0.01 ; * \star * \mathrm{P}<0.001 ; * \star \star \star P<0.0001$.
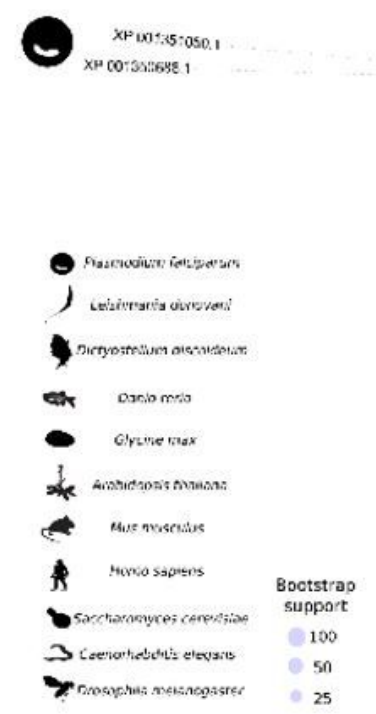

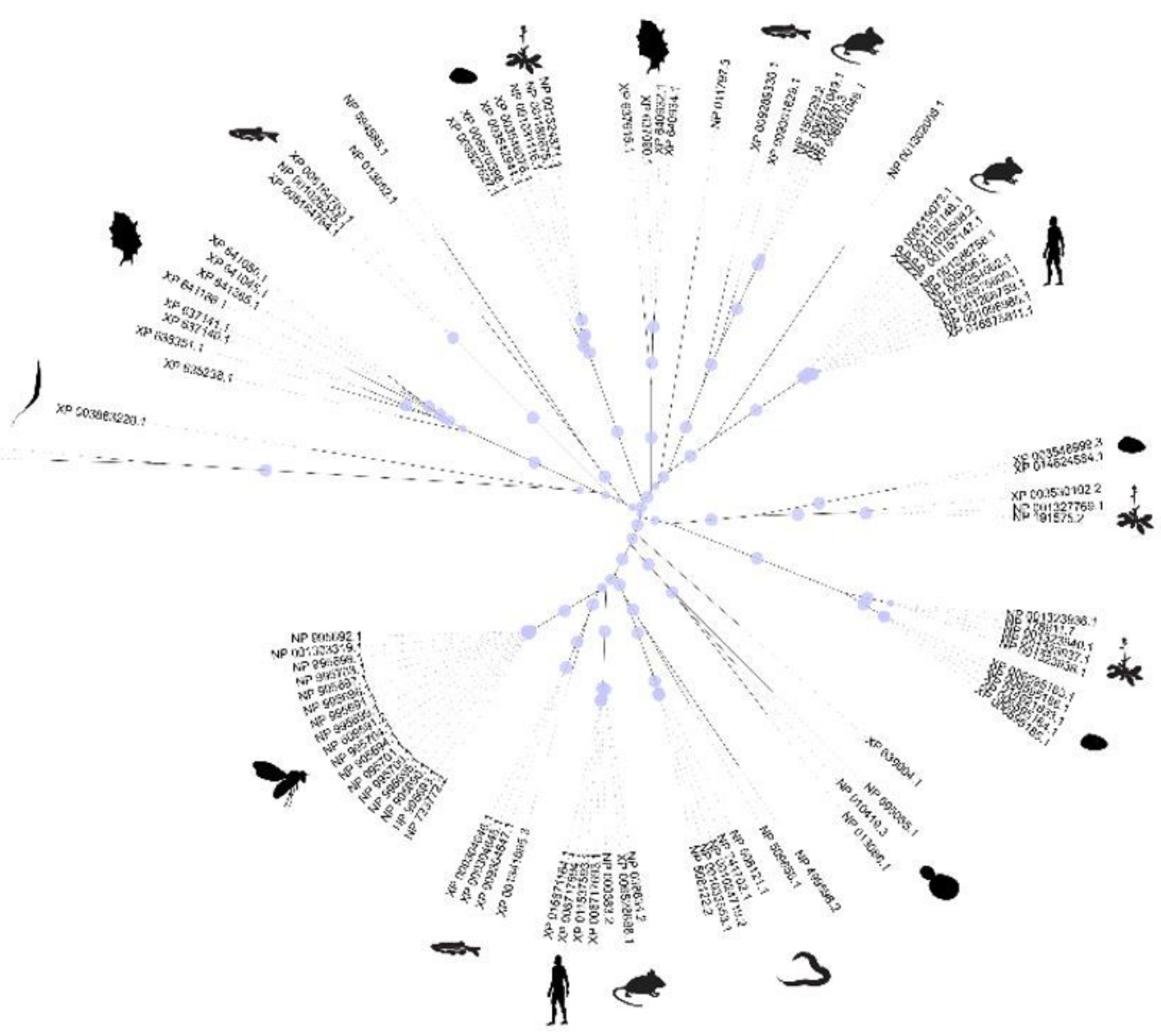

Figure 6

\section{Maximum likelihood phylogenetic representation of pfmrp1 and other $A B C$ transporters.}

Sequences were retrieved by a BLASTp query of the Landmark database using the PfMRP1 amino acid sequence. The sequences of $A B C$ transporters spanning a wide taxonomic range $(n=117)$ were aligned with MAFFT and the phylogenetic analysis was performed with IQtree. Tree visualization was done with iTOL using PhyloPic images. Bootstrap support values are indicated in each node with blue circles. 


\section{Supplementary Files}

This is a list of supplementary files associated with this preprint. Click to download.

- SupplementaryDataset1.xlsx

- SupplementaryDataset2.xlsx 